Revue musicale OICRM

\title{
La Ligne rouge de Hans Zimmer. Matrice d'un « nouvel Hollywood " électro-minimaliste et contemplatif
}

\section{Cécile Carayol}

Volume 5, numéro 2, 2018

Création musicale et sonore dans les blockbusters de Remote Control

URI : https://id.erudit.org/iderudit/1054148ar

DOI : https://doi.org/10.7202/1054148ar

Aller au sommaire du numéro

\section{Éditeur(s)}

Observatoire interdisciplinaire de création et recherche en musique (OICRM)

ISSN

2368-7061 (numérique)

Découvrir la revue

Citer cet article

Carayol, C. (2018). La Ligne rouge de Hans Zimmer. Matrice d'un « nouvel Hollywood " électro-minimaliste et contemplatif. Revue musicale OICRM, 5(2), 79-102. https://doi.org/10.7202/1054148ar
Résumé de l'article

À travers une étude comparative de plusieurs films au contexte narratif contemplatif comme La Ligne Rouge (Terrence Malick, 1998), partition-matrice qui a marqué une nette évolution dans l'esthétique zimmerienne, Hannibal (Ridley Scott, 2001), Da Vinci Code (de Ron Howard, 2006) « synthèse la plus raffinée des influences du minimalisme " (Berthomieu 2013, p. 698), jusqu’à des partitions que Hans Zimmer a composées pour Christopher Nolan comme Inception (2010) et Interstellar (2014), cet article montre de quelle manière Zimmer parvient pleinement à imposer un nouveau courant musical à Hollywood en intégrant une écriture épurée imprégnée notamment par le minimalisme d'Arvo Pärt à des boucles élaborées par des synthétiseurs ou des sons électroniques : si les hommages ciblés à des oeuvres d'Arvo Pärt sont propices à souligner le tourment intérieur ou le recueillement sombre, Zimmer reprend également des principes plus généraux de cette forme de minimalisme - souvent une oscillation immuable et répétée à l'infini autour d'un accord parfait mineur - presque systématiquement mêlés à cette énergie créative de timbres hybrides, afin de créer une autre temporalité apportant une forme d'inéluctable à l'image tout en maintenant empathie et synchronisme discret comme soutiens à l'action (La Ligne rouge, Batman Begins, Da Vinci Code, Inception). La quinte - seule, en ostinato ou répétée sur un motif - quintessence du tintinnabuli zimmerien (au-delà de l'accord parfait pärtien), souligne l'instant suspendu (La Ligne rouge, Hannibal, Interstellar), tandis qu'une forme de radicalisation de ce minimalisme qui va parfois jusqu'à la négation de toute mélodie, remplacée par une note unique, devenue texture abstraite, ou par un cluster diatonique en blend mode (Da Vinci Code, Interstellar), évoque le désespoir, la mort, ou le néant. Loin d'être un « monde " qui « se réduit alors au vide d'un présent sans rêve » (Berthomieu 2004, p. 75), l'écriture électro-minimaliste et contemplative de Zimmer, marquée par une cohérence narrative forte, est connectée au programme esthétique des films auxquels elle se destine.
Ce document est protégé par la loi sur le droit d'auteur. L'utilisation des services d’Érudit (y compris la reproduction) est assujettie à sa politique d'utilisation que vous pouvez consulter en ligne.

https://apropos.erudit.org/fr/usagers/politique-dutilisation/ 


\title{
La Ligne rouge de Hans Zimmer. Matrice d'un " nouvel Hollywood" électro-minimaliste et contemplatif
}

\author{
Cécile Carayol
}

\begin{abstract}
Résumé
À travers une étude comparative de plusieurs films au contexte narratif contemplatif comme La Ligne Rouge (Terrence Malick, 1998), partition-matrice qui a marqué une nette évolution dans l'esthétique zimmerienne, Hannibal (Ridley Scott, 2001), Da Vinci Code (de Ron Howard, 2006) «synthèse la plus raffinée des influences du minimalisme » (Berthomieu 2013, p. 698), jusqu'à des partitions que Hans Zimmer a composées pour Christopher Nolan comme Inception (2010) et Interstellar (2014), cet article montre de quelle manière Zimmer parvient pleinement à imposer un nouveau courant musical à Hollywood en intégrant une écriture épurée imprégnée notamment par le minimalisme d'Arvo Pärt à des boucles élaborées par des synthétiseurs ou des sons électroniques : si les hommages ciblés à des œuvres d'Arvo Pärt sont propices à souligner le tourment intérieur ou le recueillement sombre, Zimmer reprend également des principes plus généraux de cette forme de minimalisme - souvent une oscillation immuable et répétée à l'infini autour d'un accord parfait mineur - presque systématiquement mêlés à cette énergie créative de timbres hybrides, afin de créer une autre temporalité apportant une forme d'inéluctable à l'image tout en maintenant empathie et synchronisme discret comme soutiens à l'action ( $\mathrm{La}$ Ligne rouge, Batman Begins, Da Vinci Code, Inception). La quinte - seule, en ostinato ou répétée sur un motif - quintessence du tintinnabuli zimmerien (au-delà de l'accord parfait pärtien), souligne l'instant suspendu (La Ligne rouge, Hannibal, Interstellar), tandis qu'une forme de radicalisation de ce minimalisme qui va parfois jusqu'à la négation de toute mélodie, remplacée par une note unique, devenue texture abstraite, ou par un cluster diatonique en blend mode (Da Vinci Code, Interstellar), évoque le désespoir, la mort, ou le néant. Loin d'être un «monde » qui « se réduit alors au vide d'un présent sans rêve " (Berthomieu 2004, p. 75), l'écriture électro-minimaliste et contemplative de Zimmer, marquée par une cohérence narrative forte, est connectée au programme esthétique des films auxquels elle se destine.
\end{abstract}

Mots clés : Zimmer ; électro-minimaliste ; minismalisme ; contemplatif ; Pärt ; statique ; électronique ; synthétique ; hybride ; texture abstraite ; matrice ; épure ; radicalisation.

\section{Abstract}

Through a comparative study of several films with a contemplative narrative context such as Thin Red Line (Terrence Malick, 1998), a score-matrix that marked a clear 
evolution in Zimmerian aesthetics, Hannibal (Ridley Scott, 2001), The Da Vinci Code (Ron Howard, 2006) "the most sophisticated synthesis of the influences of minimalism" (Berthomieu 2013, p. 698), to scores that Hans Zimmer composed for Christopher Nolan as Inception (2010) and Interstellar (2014), this article shows how Zimmer manages to impose a new musical trend in Hollywood by integrating a pared-down writing impregnated notably by the minimalism of Arvo Pärt to loops developed by synthesizers or electronic sounds: if tributes to works of Arvo Pärt are appropriate to highligh inner torment or dark meditation, Zimmer also takes up more general principles of this form of minimalism - often an immutable and infinitely repeated oscillation around a minor perfect chord - almost systematically mixed with this creative energy of hybrid timbres, to create another temporality bringing a form of ineluctable to the image while maintaining empathy and discreet synchronism as supports for the action (The Thin Red Line, Batman Begins, The Da Vinci Code, Inception). The fifth - alone, in ostinato or repeated on a motif -, which is the quintessence of the Zimmerian tintinnabuli (beyond the perfect Pärtian agreement), emphasizes the suspended moment (The Thin Red Line, Hannibal, Interstellar), while a form of radicalization of this minimalism which sometimes goes as far as the negation of any melody, replaced by a single note, becoming abstract texture, or by a diatonic cluster in blend mode (The Da Vinci Code, Interstellar), evokes despair, death, or nothingness. Far from being a "world" which "is reduced to the emptiness of a present without a dream" (Berthomieu 2004, p. 75), Zimmer's electro-minimalist and contemplative writing, marked by a strong narrative coherence, is connected to the aesthetic program of the films for which it is intended.

Keywords: Zimmer; electro-minimalism; minismalism; contemplative; Pärt; static; electronic; synthetic; hybrid; abstract texture; matrix; outline; radicalization.

Si Hans Zimmer ne déroge pas forcément aux codes instaurés par les compositeurs du néo-hollywoodisme ${ }^{1}$, cet article s'applique à montrer en quoi sa manière de privilégier et d'intégrer une forme d'épure dans son écriture (imprégnée notamment par le minimalisme d'Arvo Pärt) à des boucles élaborées par des synthétiseurs ou des sons électroniques, participe pleinement à imposer un nouveau courant musical à Hollywood à l'aube de ce vingt et unième siècle, à apporter des significations narratives différentes et à transporter la perception du rapport musique-images dans une temporalité autre.

Afin de cerner l'un des aspects majeurs du langage de ce compositeur, cette étude s'appuie sur l'analyse de plusieurs films "d'émotions collectives " (Chion 1995, p. 155) comportant une dimension contemplative plus ou moins marquée comme La Ligne rouge (Terrence Malick, 1998), dans une moindre mesure Hannibal (Ridley Scott, 2001) et Batman Begins (premier volet de la trilogie de Christopher Nolan, 2005), ou encore Da Vinci Code (Ron Howard, 2006), Inception (Christopher Nolan, 2010) et Interstellar (Christopher Nolan, 2014). Elle se fonde en partie sur les pistes

\footnotetext{
1 Au sujet du néo-hollywoodisme, voir par exemple Carayol 2012 (chapitre II). La partition de Hans Zimmer pour Hannibal de Ridley Scott (2001) s'inscrit en partie dans le prolongement des principes musicaux du cinéma d'horreur des années 1990.
} 
amorcées par Pierre Berthomieu dans plusieurs de ses ouvrages, quelques études consacrées à Arvo Pärt, ainsi que sur quelques-uns de nos travaux précédents sur le symphonisme intimiste et la dimension contemplative et minimaliste de la musique dans le film de guerre à travers l'exemple de La Ligne Rouge qui a servi de matrice ici ${ }^{2}$. Ce film qui « repose sur des idées minimalistes, déclinées et enflées par des nappes synthétiques voluptueuses sur de longues durées " (Berthomieu 2004, p. 140), auquel on relie souvent le son Zimmer et Remote control $^{3}$, marque une rupture stylistique par rapport à ce qui se pratiquait auparavant, ouvrant une voie nouvelle dans la musique du cinéma hollywoodien.

Après avoir observé en quoi les hommages ciblés à des œuvres d'Arvo Pärt sont propices à souligner le tourment intérieur ou le recueillement sombre, l'analyse se centrera sur trois notions issues de principes plus généraux de ce minimalisme statique : le brouillage des repères temporels par les nappes électro-minimalistes, la quinte répétée comme symbole de l'instant suspendu, ou encore l'anti-lyrisme et les clusters diatoniques en "blend mode ${ }^{4}$ - sorte de radicalisation de ce minimalisme pour évoquer le désespoir, la mort ou le néant.

\section{«Darkness into Light » - Hommages À Arvo PÄrt :}

\section{TOURMENT INTÉRIEUR ET RECUEILLEMENT SOMBRE}

Le film La Ligne rouge traite de la bataille de Guadalcanal dans le Pacifique opposant les Américains aux Japonais pendant la Seconde guerre mondiale. Terrence Malick livre une réflexion métaphysique de l'homme face à la guerre, son rapport à l'amour, à la mort et à la nature, à travers les monologues intérieurs de soldats qui finissent par se confondre. Il filme la beauté d'une nature paisible par de nombreux plans méditatifs et poétiques qui exacerbent d'autant plus la violence des combats. Pour répondre à ce choix esthétique, la musique de Zimmer offre un contrepoint aux champ de batailles avec une écriture minimaliste qui s'exprime notamment à travers l'inspiration du compositeur estonien. Zimmer en retient surtout la dimension contemplative, mais parfois cette influence permet aussi d'accompagner des situations où le sacré, et du moins le caractère spirituel, a une place prépondérante dans le récit. La séquence de La Ligne rouge où les soldats américains sont en période de relâche sur l'île - moment propice aux réflexions, aux introspections secrètes, ou encore au remord qui consume l'être, Zimmer écrit un motif au titre évocateur " Light " qui est une réminiscence du Cantus in memoriam Benjamin Britten en la mineur modal (extrait vidéo 1 et extrait audio 1). On entend l'esquisse de la même référence dans le cue «Virtue » de Hannibal

2 Pour toutes ces références, voir la bibliographie en fin d'article.

3 Anciennement nommé Media Ventures, Remote Control Productions est un studio fondé par Hans Zimmer en 2003 comprenant des compositeurs à l'image (James Newton Howard, Klaus Badelt, Rupert GregsonWilliams, John Powell, etc.) avec lesquels il lui arrive de partager le travail sur une bande originale et d'autres collaborateurs liés plus ou moins directement à la conception musicale et sonore d'un film. Remote Control comporte des studios de mixage, d'enregistrement et produit également des films, des sagas et des jeux vidéo.

4 Orchestration hybride mêlant instruments acoustiques et sonorités de synthèse (électroniques, numériques). 
dans un contexte plus postromantique au moment où le Docteur Lecter soigne l'agent Starling, dont il est épris, pour accompagner cette brève trêve du monstre. Mais c'est surtout dans le cue de Da Vinci code intitulé "Malleus Maleficarum " (marteau des sorcières) au caractère contemplatif, mystique et douloureux que cet hommage au Cantus réapparaît ostensiblement pour souligner un des flashbacks les plus importants du film, dans lequel le secret sur la descendance du Christ nous est révélé par des images évocatrices de peintures préraphaélites (extrait audio 2 et figures $1 \mathrm{a}, 1 \mathrm{~b}$ et $1 \mathrm{c}$ ). Que ce soit dans «Light » ou " Malleus Maleficarum », une même ligne mélodique à la mélancolie recueillie chute inlassablement, donnant une impression similaire de géotropisme statique fait essentiellement de valeurs longues. Le tout converge sur une gamme modale et non modulante - do mineur modal dans La Ligne Rouge, la mineur modal dans Da Vinci Code - renvoyant à la même sacralisation de 1'accord parfait ${ }^{5}$ (exemples $1 \mathrm{a}$ et $1 \mathrm{~b}$ ).

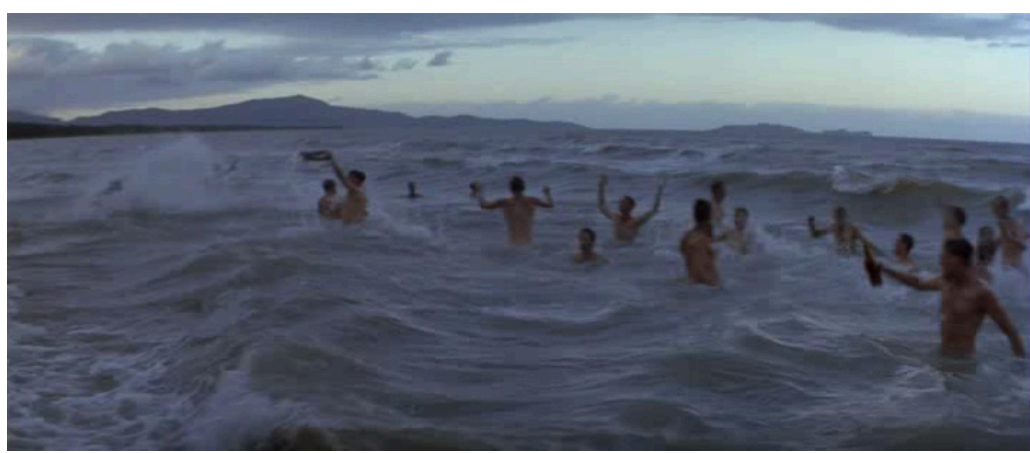

Extrait vidéo 1 : Terrence Malick, La Ligne rouge, recueillement sombre des soldats pendant une période de relâche (02:00:43- $02: 03$ :23) () Twentieth Century Fox Film Corporation.

Extrait audio 1 : Arvo Pärt, Cantus firmus in memoriam Benjamin Britten (00:36-02:18)

(C) Bis Records 1997. Écouter.

Extrait audio 2 : Hans Zimmer, Da Vinci Code, cue «Malleus Maleficarum»(00:00-00:57) (C) Decca. Écouter.
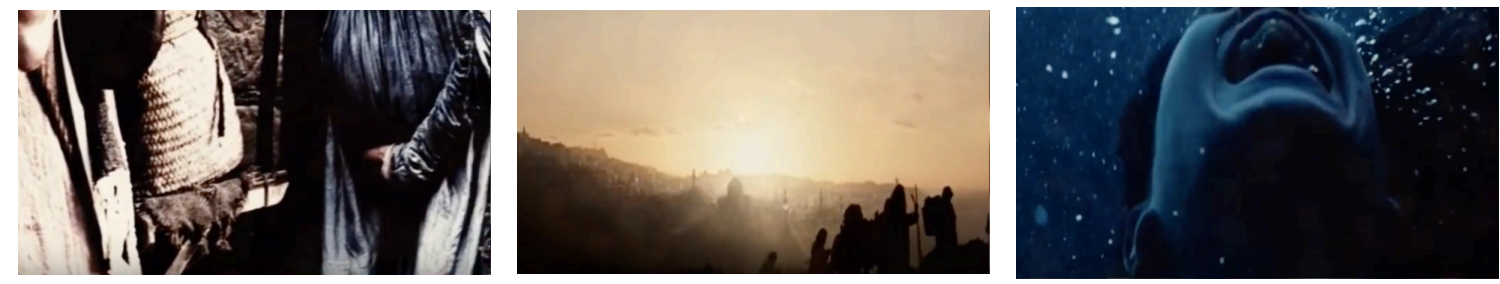

Figures 1a, 1b et 1c: Ron Howard, Da Vinci Code, trois photogrammes du flashback de la scène de Marie-Madeleine enceinte et de la légende du marteau des sorcières (01:08:10-01:10:15) (c) Colombia Pictures.

5 Arvo Pärt explique : «J'ai découvert que c'était suffisant lorsqu'une seule note était bien jouée. Cette note, ou une pulsation de silence, ou un moment de silence me réconfortent. Je travaille avec très peu d'éléments - avec une voix, avec deux voix. Je construis avec les matériaux les plus primitifs - avec l'accord parfait, avec une tonalité spécifique. Trois notes d'un accord sont comme des cloches. Et c'est pourquoi j'appelle cela tintinnabulation » (propos recueillis par Wolfgang Sandner pour Tubula Rasa, dans Moindrot 2004, p. 69). 

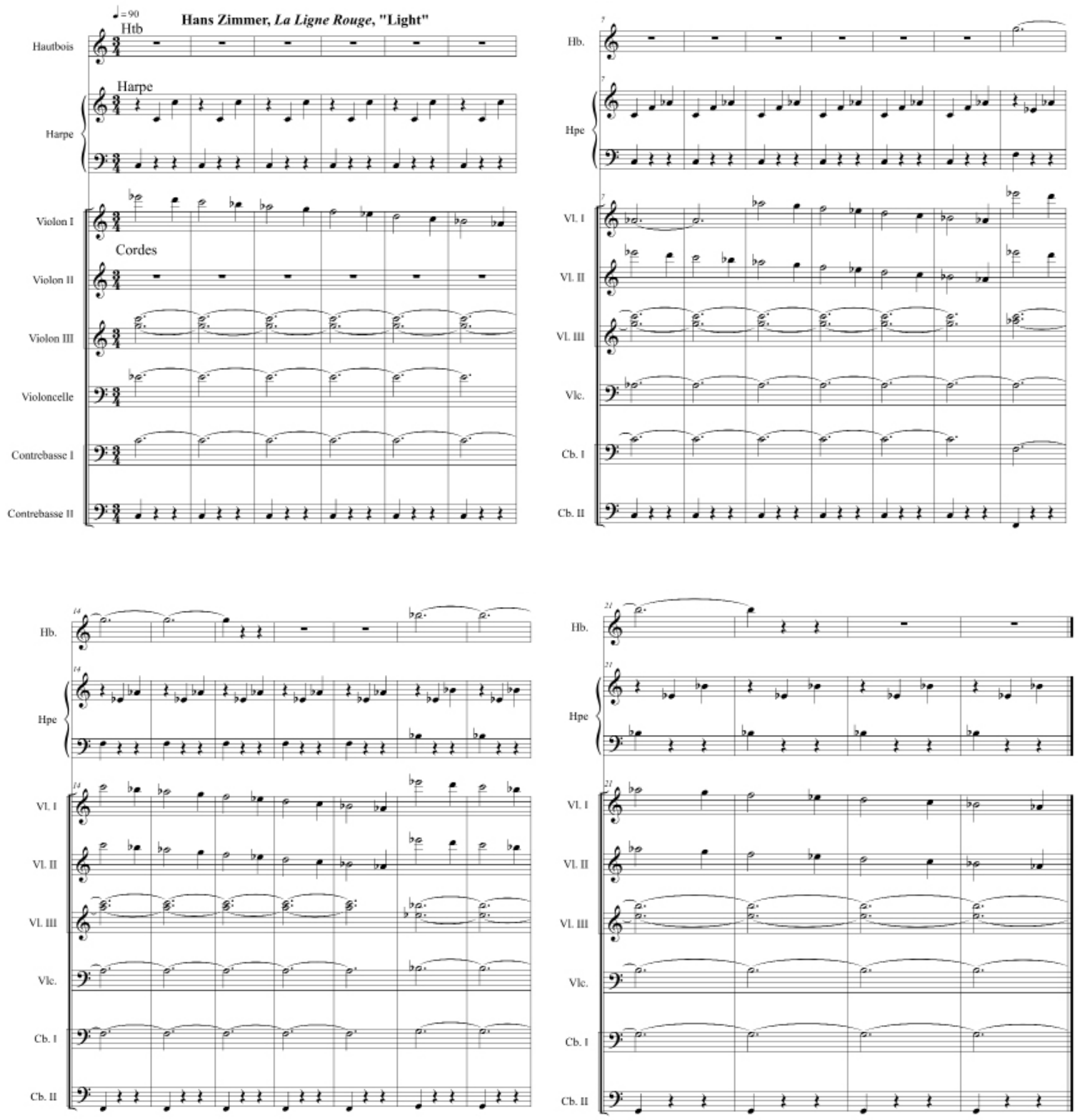

Exemple 1a: Hans Zimmer, La Ligne rouge, cue "Light». Transcription personnelle (mes. 1-13), à partir de la bande originale (03: 46-04:41). 

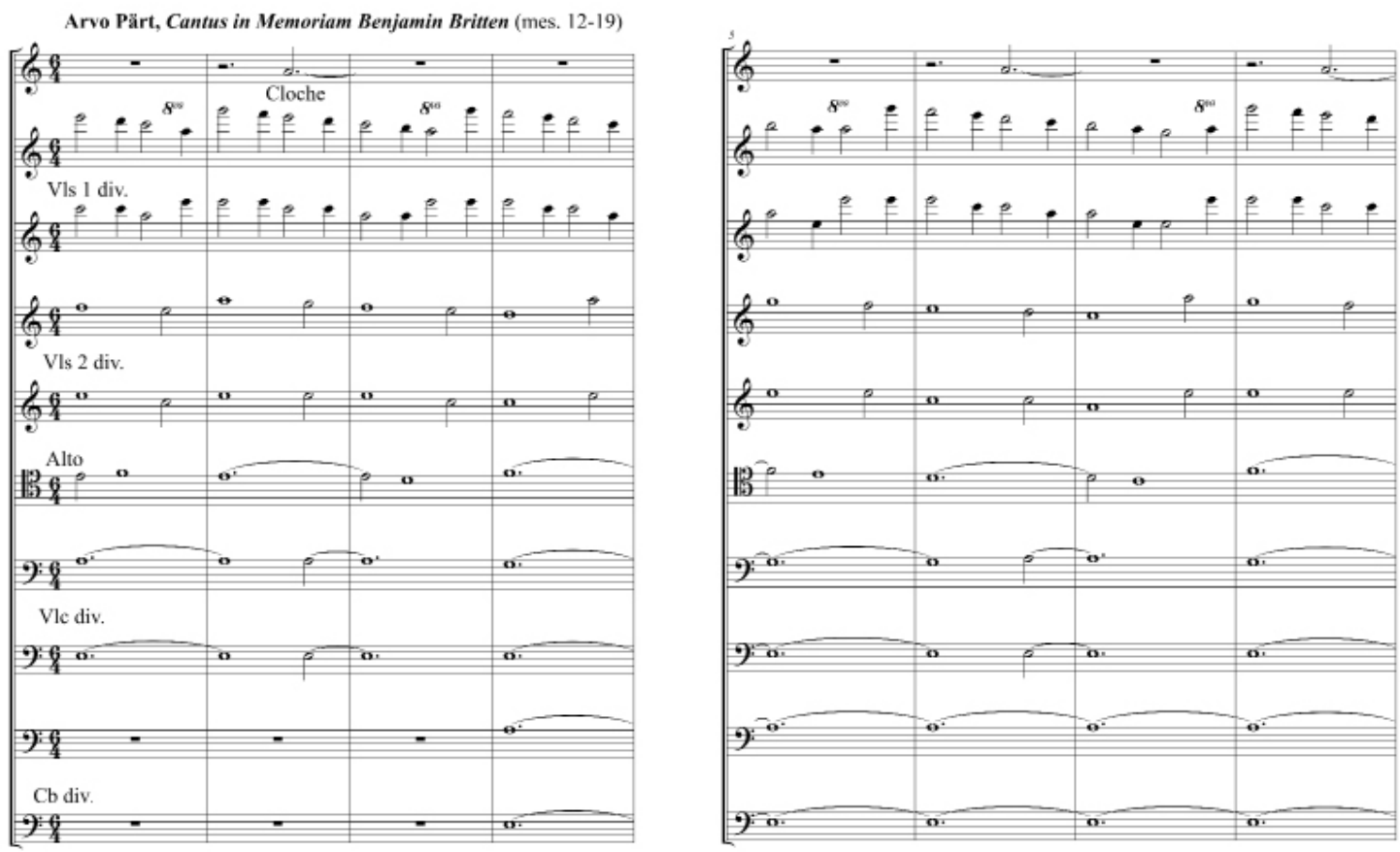

Exemple 1b: Arvo Pärt, Cantus firmus in Memoriam Benjamin Britten (mes. 12-20).

Transcription personnelle à partir $d u C D^{6}$.

Dès la première apparition de Silas dans Da Vinci Code, moine sombre de l'Opus Dei, Zimmer compose un cue intitulé "The Paschal Spiral " qui se rapproche en grande partie de Festina Lente et de Trisagion de Pärt (œuvre très symbolique évoquant la trinité) pour souligner les châtiments corporels que s'inflige à deux reprises ce personnage à la fois bourreau et martyr semblant donner vie à certaines peintures de la renaissance lorsqu'il serre un Silice sur sa cuisse ensanglantée, puis quand il fouette son dos (figures $2 \mathrm{a}, 2 \mathrm{~b}$ et $2 \mathrm{c}$, extraits audio 3,4 et 5 ).

Dans ces trois morceaux cités - «Light », « Malleus », « Paschal Spiral »-Zimmer retient de ces œuvres une même couleur instrumentale globale (violons divisés, altos, violoncelles, contrebasses) - excepté pour " Malleus " dans lequel le chœur lié à la dimension sacrée de l'histoire domine, une écriture modale, sans modulation, des valeurs allant de la noire, à la blanche pointée ou à la ronde, l'insistance sur un accord, voire une note (le mi dans "The Paschal Spiral»), des renversements de l'accord parfait invitant au suspensif - chiffré 6 dans «Light » et « Malleus » comme au début de Trisagion, ou accord de la chiffré 6/4 dans "The Paschal Spiral » comme dans le Cantus. Zimmer retient aussi le principe de "dissonance diatonique " (Moindrot 2004, p. 69) amenée soit par surimpression de deux accords ou par les notes de passages, celles de la mélodie qui chute perpétuellement ("Light ", " Malleus ») ou celle des différents contrechants ("The Paschal Spiral »). Le morceau " Malleus Maleficarum », en la mineur modal, offre une illustration convaincante de ce procédé (que l'on trouve 
dans des pièces comme le Cantus in memoriam Benjamin Britten - exemple $1 \mathrm{~b}$ - ou Trisagion). Les accords de ré mineur, de fa majeur ou de do majeur s'entremêlent à l'accord de la mineur sous forme de tuilage. Tous ces accords - harmonies blanches ne comportant aucune altération - invitent au diatonisme. Par exemple, les notes si (mesure 2) sol et $f a$ (mesure 3 ) de la mélodie qui descend de manière conjointe viennent ajouter une tension à l'accord parfait de la mineur sans heurt (tout frottement est globalement entendu comme une couleur de $9^{\mathrm{e}}$ ou de $7^{\mathrm{e}} \mathrm{d}^{\prime}$ espèce) ; les notes de l'accord de la mineur, mesures 4 et 8 , viennent, au détour d'un accord de passage, se mêler à l'accord de ré mineur (exemple 2) : "Ainsi, un fa et un ré exprimés conjointement avec un accord de la mineur forment-ils une dissonance diatonique ( (ibid., p. 69).

Pour « Paschal Spiral », comme le fait Arvo Pärt dans le début de Trisagion, Zimmer maintient une note pédale dans le grave sous une texture éthérée. Enfin, au moment précis où Silas prononce les mots "Je châtie mon corps », devant la croix du Christ fixée au mur de sa chambre ascétique, il reprend la même structure que la fin de Festina Lente. La répétition pianissimo d'un intervalle tendu à la texture fantomatique est suivie d'un silence, d'un " presque rien » (Jankélévitch 1976, p. 21) chez Zimmer, puis d'un éclat douloureux des cordes (exemples $3 a$ et $3 b$ ). Pour correspondre au programme esthétique du film qui consiste à conjuguer des éléments du passé avec un savoir faire moderne à l'aide de l'électronique et du numérique, comme ailleurs dans certaines œuvres du compositeur estonien (Cantus in memoriam Benjamin Britten, Trisagion), lequel a étudié la monodie grégorienne, Zimmer mêle ici une écriture contemporaine, mâtinée de drones et autres infrabasses, avec des hommages à la musique ancienne. Il traite un des thèmes principaux du film relié à la quête du Saint Graal - très évocateur d'un plain-chant joué à l'alto ici - à la manière d'un organum, les notes contre notes formant souvent des intervalles de quintes et de quartes (exemple 4).
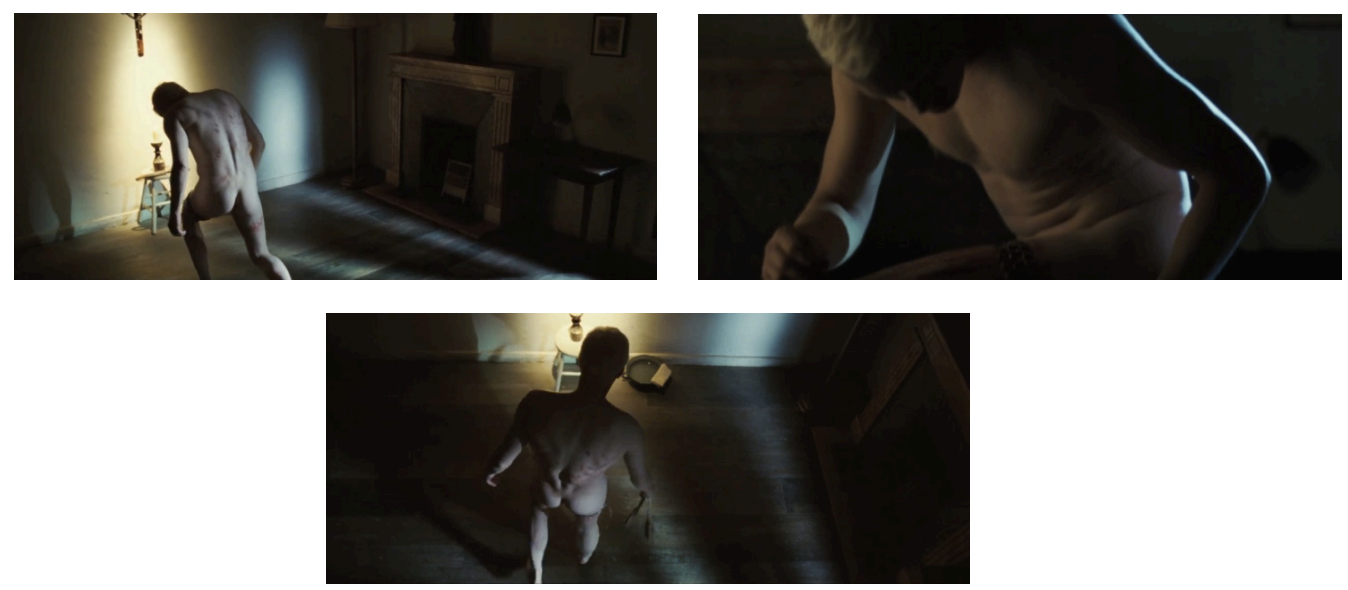

Figures 2a, 2b et 2c: Ron Howard, Da Vinci Code, trois photogrammes de la scène où Silas s'inflige des sévices corporels (07:16-08:50) (c) Colombia Pictures.

Extrait audio 3 : Hans Zimmer, Da Vinci Code, cue "The Paschal Spiral»(00:55-02:38) () Decca. Écouter.

Extrait audio 4: Arvo Pärt, Trisagion (01:39-01:57) ( Decca. Écouter.

Extrait audio 5 : Arvo Pärt, Festina Lente (04:59-05:35) (C) Decca. Écouter. 


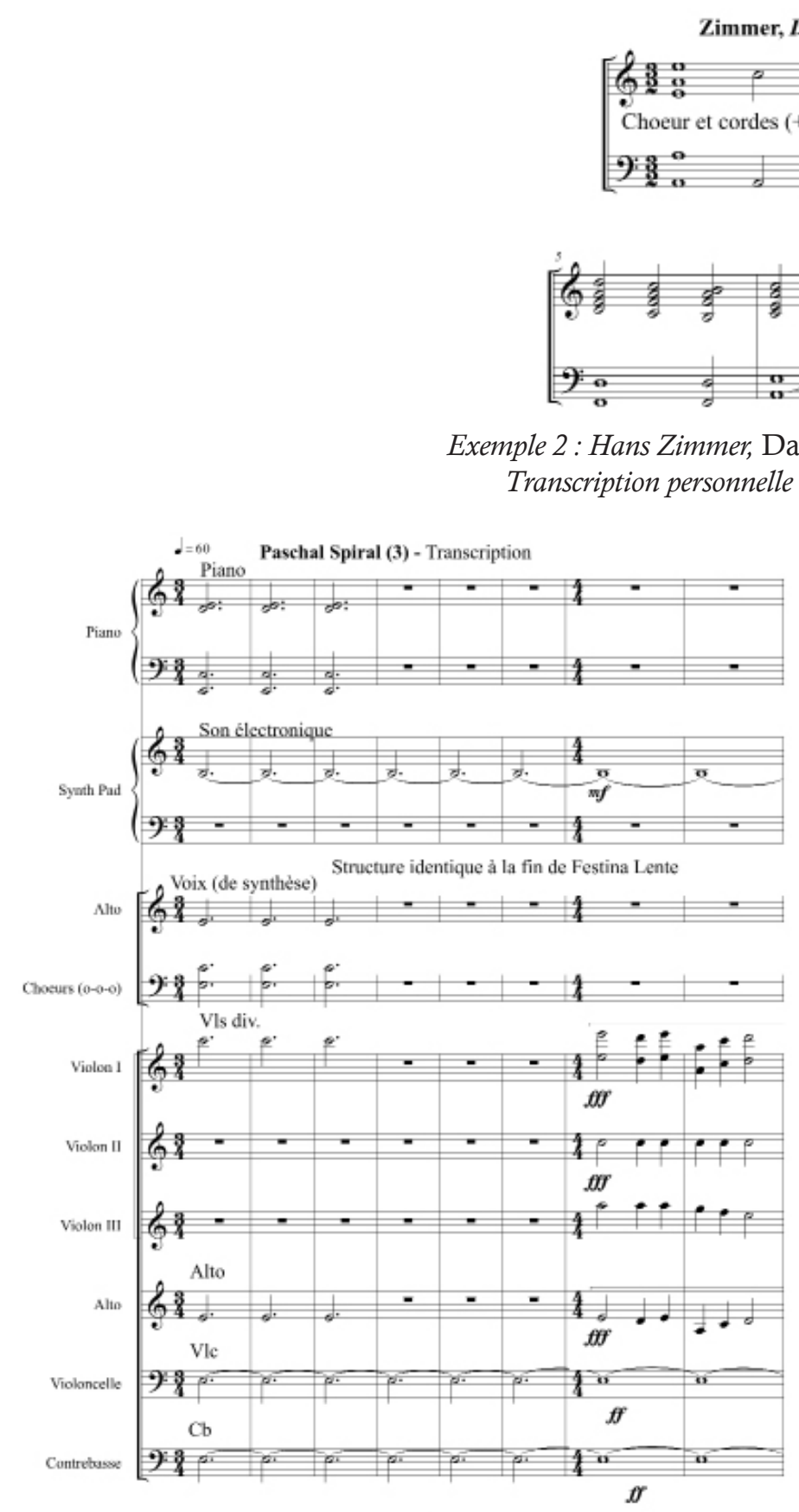

Exemple 3a: Hans Zimmer, Da Vinci Code, cue "The Paschal Spiral ». Transcription personnelle à partir de la bande originale (01:49-02:15). 

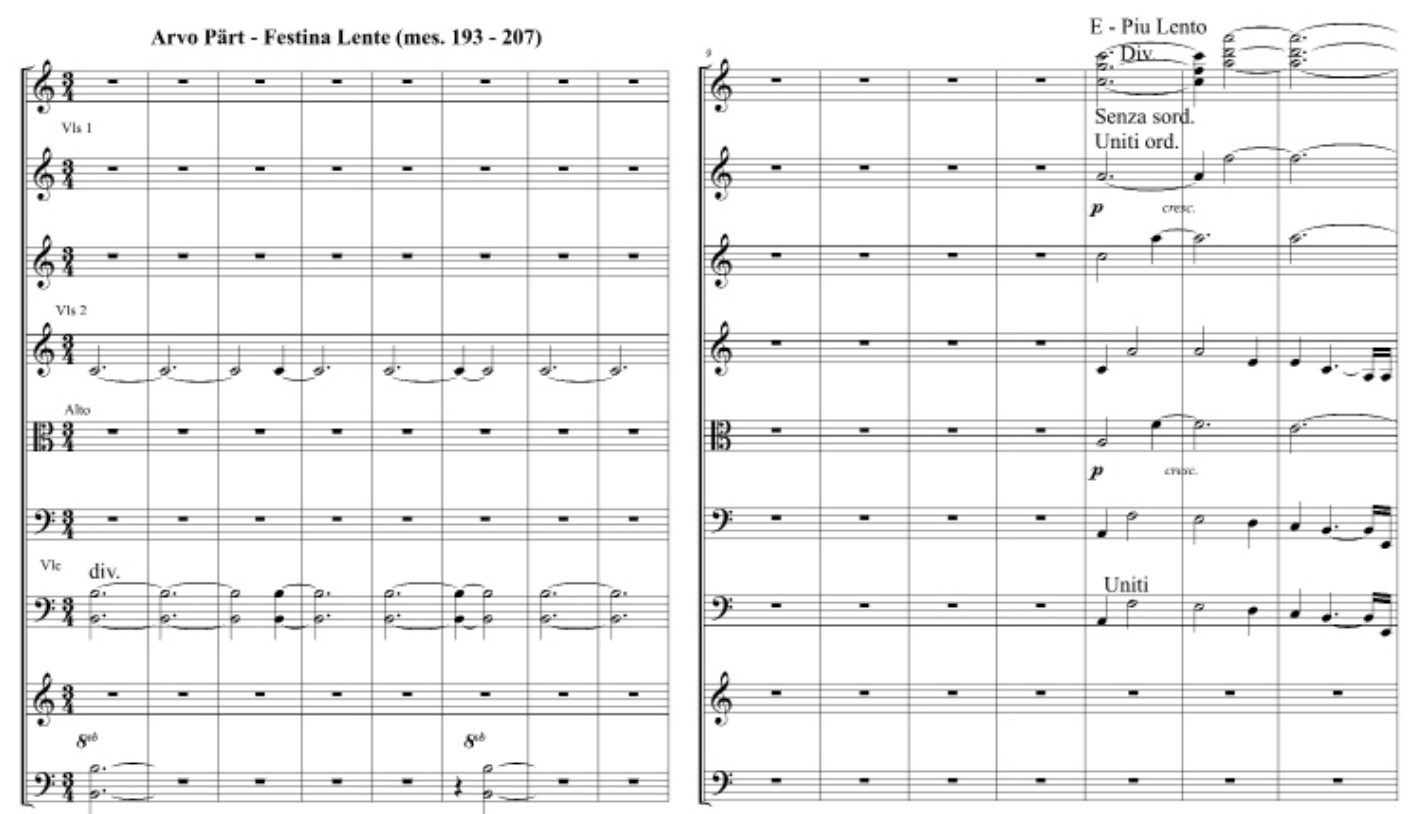

Exemple 3b : Arvo Pärt, Festina lente (mes. 193-207). Transcription personnelle à partir du $C D^{7}$.

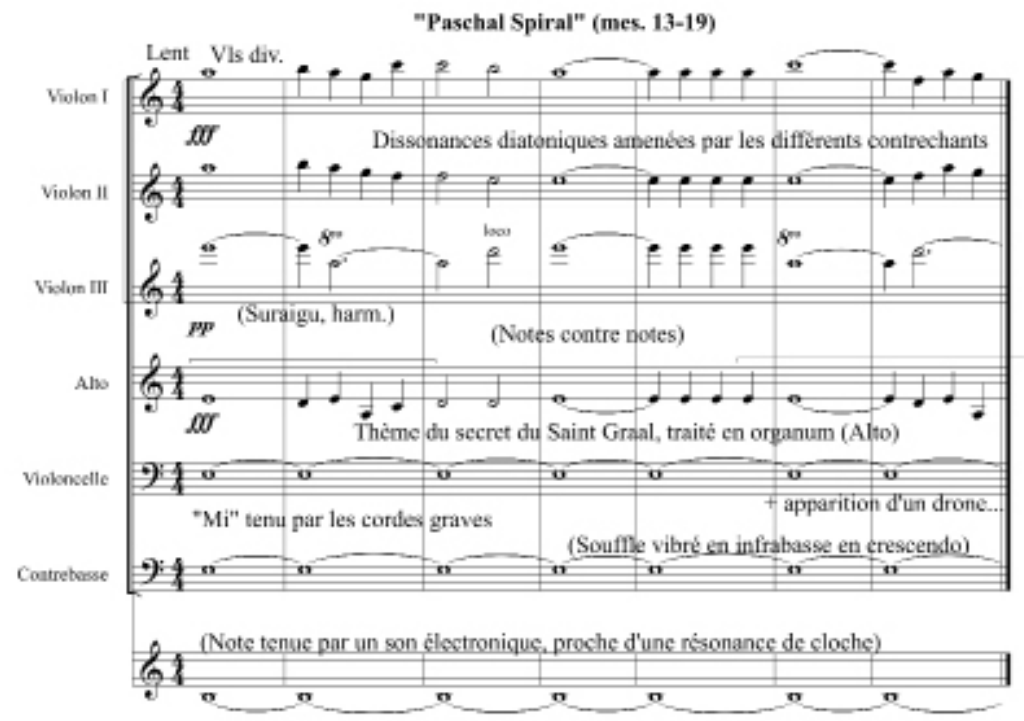

Exemple 4 : Hans Zimmer, Da Vinci Code, cue "The Paschal Spiral».

Transcription personnelle à partir de la bande originale (00:55-01:26).

LA « QUESTION SANS RÉPONSE » - UNE VARIANTE DE L'ÉPIQUE ET DE L'HÉROÏSME : BROUILLAGE DES REPÈRES TEMPORELS PAR LES NAPPES ÉLECTRO-MINIMALISTES

Même quand ce ne sont pas des hommages aussi ciblés à des œuvres précises d'Arvo Pärt, Zimmer conserve les principes généraux de ce minimalisme, produisant 
" des effets de stase [...] sur un mode plus strictement technique [c'est à dire des samples ou des boucles élaborés par des synthétiseurs et des sons numériques] que ses modèles historiques ou mystiques [Arvo Pärt en l'occurrence] " (Berthomieu 2013, p. 697). A l'instar du war adagio de La Ligne rouge (analysé dans le paragraphe suivant), d'autres partitions de Zimmer composées pour des grandes productions marquantes du cinéma hollywoodien contemporain comme Batman Begins ("Vespertilio "amorce du thème principal développé dans la trilogie), Da Vinci Code ("Chevaliers de Sangreal »), Inception (« Time») ou Interstellar ( Day One » ou " Cornfield Chase ») suivent la même trajectoire. En remodelant timbres et intervalles associés au caractère épique ou héroïque dans les bandes originales néo-hollywoodiennes, ces nappes électro-minimalistes étirées longuement en proposent une variante en procédant à un brouillage temporel dans ces films, en particulier celui de Malick ou encore ceux de Nolan (Inception, Interstellar, et plus récemment dans Dunkerque, 2017), où la notion du temps est centrale et revisitée.

Le cue "Time » entendu précisément au moment où Cobb est censé revenir au niveau 0 de la réalité pour retrouver ses enfants à la fin d'Inception, après s'être enfoncé dans plusieurs niveaux de rêves, est très semblable au war adagio que Zimmer - connu depuis pour ses créations de timbres hybrides - avait écrit pour la séquence de l'assaut du camp japonais par les soldats américains dans La Ligne Rouge 8 . Le war adagio étant une variation épurée, moderne et personnelle de celui de Samuel Barber retenu pour Platoon. Dans les deux films, ces nappes cycliques dont le statisme est exacerbé ici par la modalité (Fleury 1996, p. 219) - ré mineur modal pour La Ligne rouge, la mineur modal pour Inception - évoluent de façon identiques. Une trame minimaliste - cycle de onze mesures dans La Ligne rouge et cycle de huit mesures dans Inception - fait entendre une succession de tierces en valeurs longues écrasées dans le grave pour souligner le caractère tragique de la guerre ou éclatées alternativement en $10^{\mathrm{e}}$ pour symboliser les projections mentales de Cobb. Ces cycles sont pris dans un gonflement pyramidal marqué par la fusion de sonorités traditionnelles (cordes, cuivres, percussions, voire le piano dans Inception) avec des timbres actuels (synthétiseurs, infrabasses, sons numériques, voire la guitare électrique dans Inception). Des contrechants en noires au mouvement descendant et une pulsation émergent peu à peu à l'intérieur de ces motifs extatiques. Cela crée à la fois une empathie émotionnelle et un synchronisme " aléatoire" (Berthomieu 2004, p. 137) associé à la mort guerrière (par exemple la cloche plus ou moins synchrone avec les tirs) ou au rêve potentiellement inaccessible de Cobb (celui de retrouver enfin ses enfants).

À la fin de l'affrontement des deux camps ennemis dans La Ligne rouge, les cordes aiguës divisées de la Question sans réponse (première contemplation - au titre évocateur) de Charles Ives se superposent en clair-obscur au monochrome sombre du war adagio pour relier agonie et élégie ou souffrance et déshumanisation. Selon Michel Guiomar,

$8 \quad$ Pour le film Les Associés (Ridley Scott, 2003), Zimmer reprend pratiquement le même motif en moins étendu que celui de La Ligne rouge. 
le clair-obscur se présente comme un jeu réciproque de contre-jour et de contre-nuit, [...] équilibre statique de deux équilibres dynamiques établis, [...] neutralisation qui se révèlera comme l'un des caractères essentiels du Funèbre (Guiomar 1988, p. 79).

Pour autant, les deux morceaux entretiennent également une réelle parenté. Ils sont connectés tout d'abord par la structure : le réalisateur a fait le choix de donner la forme ABA au morceau de Ives - symétrie absente dans l'œuvre d'origine - afin de correspondre à la forme en arche du war adagio. On y trouve aussi un minimalisme statique similaire en rondes et valeurs longues. Le contrechant en noires du violoncelle de la Question sans réponse semble être un écho élégiaque du war adagio, comme si le morceau de Zimmer résonnait encore, même de manière disloquée et distendue, dans le morceau de Ives. Que ce soit la mélodie disjointe de la trompette solo atonale de la Question sans réponse (dont le commentaire strident des bois dans l'œuvre originale ont été retirés) ou les cuivres du war adagio bouchés puis sans sourdine - mais doublés par le synthétiseur pour homogénéiser leur brillance - les deux morceaux, aux allures d'hymnes contrariés, ne louent pas le courage des soldats. L'un murmure un héroïsme discret, l'autre déplore, avec une sorte d'ironie amère, ce champ funeste de désolation où les corps sans vie sont déjà convoités par les vautours. En créant une osmose dans son écriture avec celle du morceau de Ives, bien plus qu'une " grande question déconnectée » (Chion 2004, p. 35), Zimmer estompe surtout la limite entre héroïsme et folie des soldats. À la fin de La Ligne Rouge, à la place d'un hymne héroïque qui aurait pu accompagner les soldats survivants traversant le cimetière militaire, on entend le spectre sombre et minimaliste du war adagio (cue intitulé "Silence ») - hymne évité. C'est un "Silence " élégiaque de la musique qui correspond à la partie en intervalles creux du war adagio (vide funeste), avec la ponctuation de la cloche tel un glas, en guise d'un dernier adieu aux soldats morts au combat.

La musique crée une sorte de temporalité oxymore entre empathie émotionnelle et distance. Dans La Ligne rouge, elle semble absorber la violence des coups, les blessés agonisant, le montage très morcelé, les effets de caméra à l'épaule, les panoramiques latéraux vertigineux, ou encore les sonorités guerrières, intégrant les bruitages en un tout coagulé afin de souligner le caractère implacable de l'assaut. Dans Inception, lequel donna à Zimmer le " nouveau défi d'une formule susceptible de traiter à la fois la matière du temps et le concept du film qui bascule personnages et public d'un univers mental à l'autre " (Berthomieu 2013, p. 698), elle devient l'alliée de Cobb, lui insufflant une sorte d'énergie qui l'encourage, que ce soit réel ou non, à ne pas cesser de croire qu'il va enfin retrouver ses enfants, alors que la mise en scène montre à quelques reprises le doute qui s'immisce dans son esprit : regard perplexe de Cobb ou personnages qu'il croise (dont son beau-père) ponctuellement floutés. Les deux morceaux ont la même forme en arche, commençant et terminant par la " silhouette " du motif - tierces graves sous un cliquetis électronique dans La Ligne rouge, partie supérieure de la mélodie jouée par les harmoniques de cordes dans Inception - pour accompagner un même type de plans en début et fin de séquence : soldats agonisants ou morts et à demi enterrés dans La Ligne rouge ; la toupie symbole possiblement illusoire (comme la bague de mariage) censée indiquer si Cobb se trouve dans un niveau de rêve ou non dans Inception. 


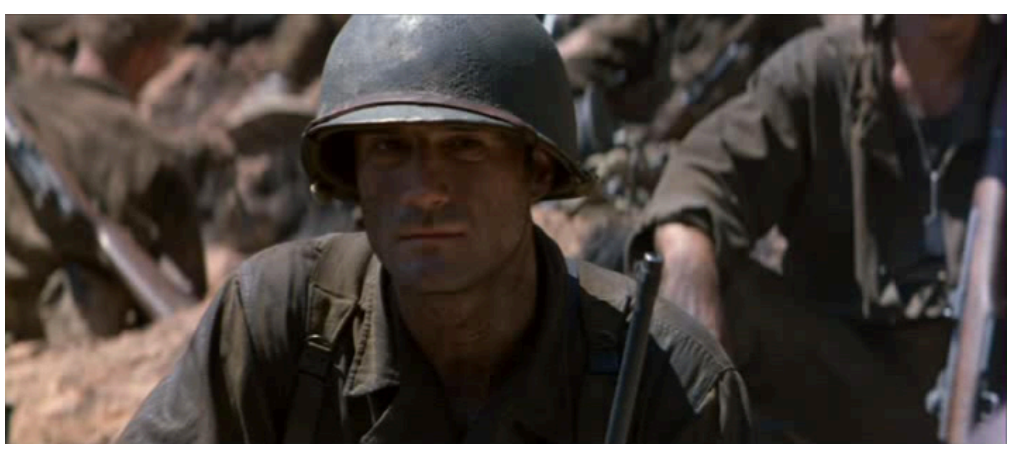

Extrait vidéo 2: Terrence Malick, La Ligne rouge, séquence de l'assaut du camp japonais par les soldats américains (01:39:36-01:50:18) (C) Twentieth Century Fox Film Corporation.
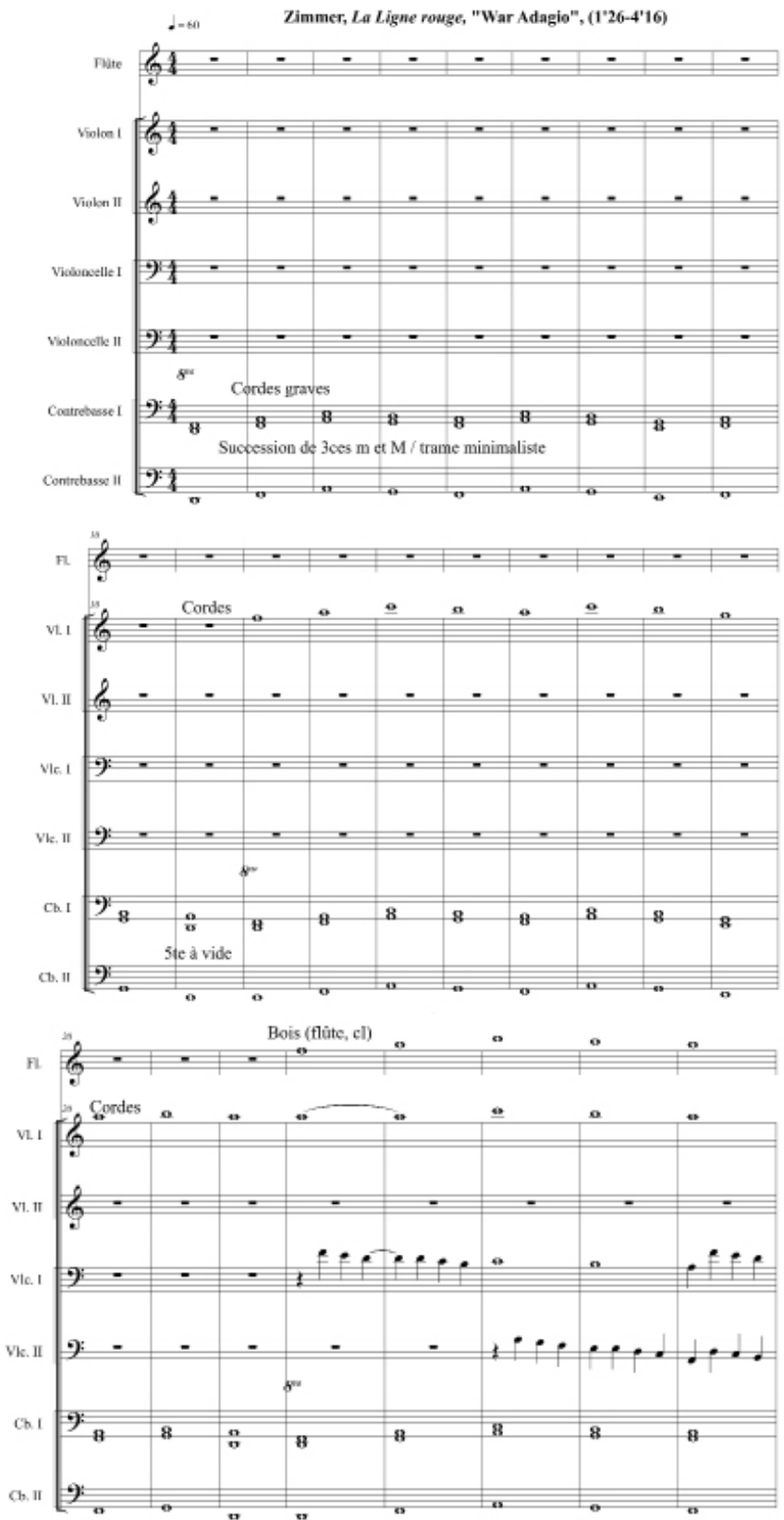

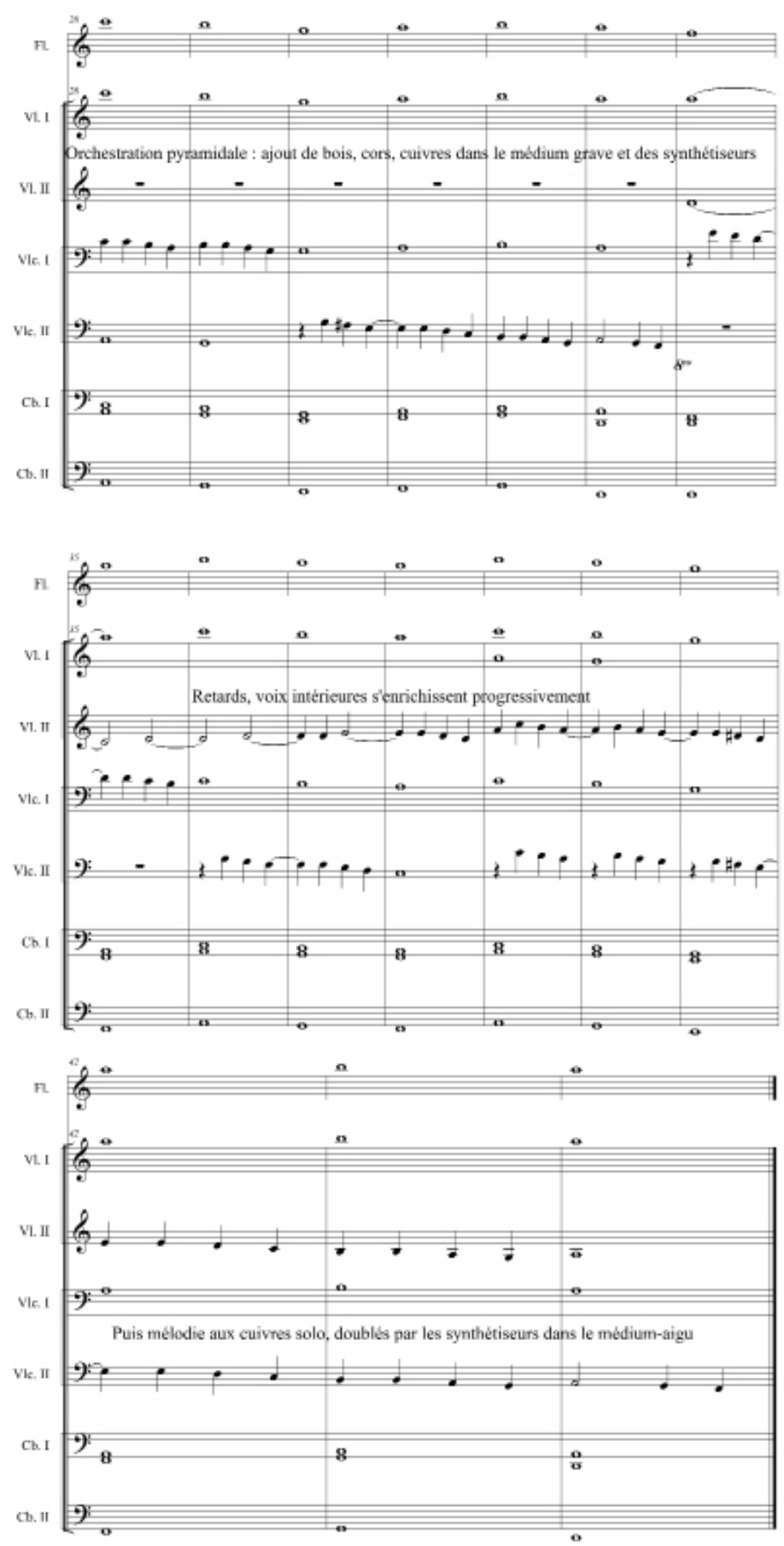

Exemple 5 : Hans Zimmer, La Ligne rouge, cue "War Adagio ». Transcription personnelle à partir de la bande originale (fragments).

Extrait vidéo 3 : Christopher Nolan, Inception, scène du retour éventuel de Cobb à la fin du film (02:11:04-02:15:06)

(C) Warner Bros. Pictures.

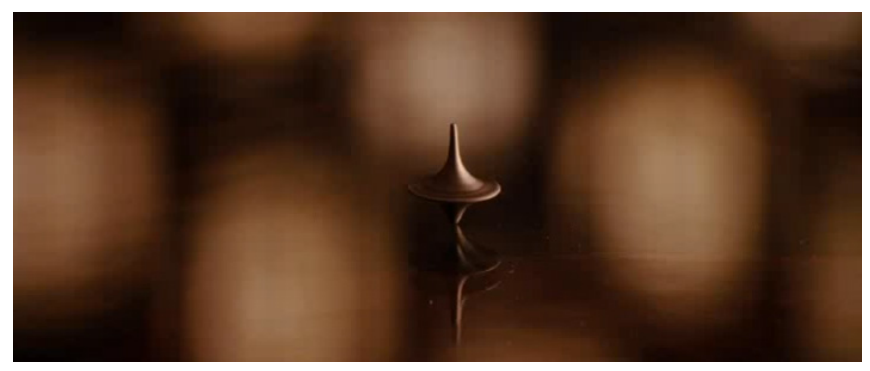



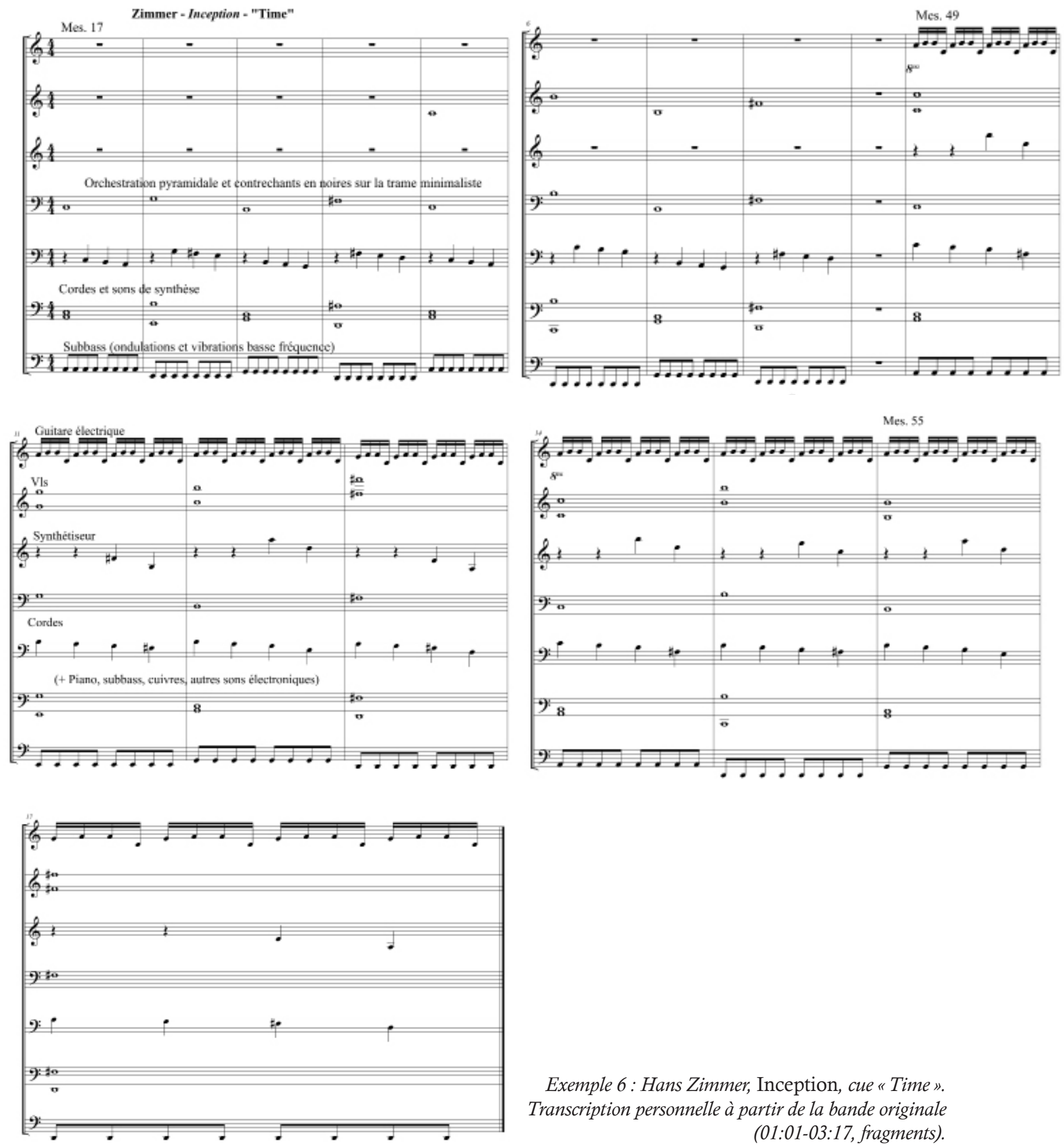

Exemple 6 : Hans Zimmer, Inception, cue "Time». Transcription personnelle à partir de la bande originale (01:01-03:17, fragments).

Le thème associé au héros Batman («Vespertilio ») dès le premier épisode de la trilogie (Batman Begins, Nolan, 2005), que Zimmer co-écrit avec James Newton Howard, s'inscrit dans un schéma à peu près similaire à celui du war adagio de La Ligne rouge. Une même série de cellules - moins statique, les doubles remplaçant les rondes - 
forme une boucle autour de l'accord parfait ré mineur (modal) étiré sur un temps long. Cela est vrai pour la trilogie entière et particulièrement prégnant dans The Dark Knight Rises, 2015. Le motif évolue peu à peu par l'ajout de strates sonores pour devenir très massif selon une convention du genre : après un battement d'aile (sound design) sur une infrabasse réalisée à l'aide de distorsion de basse (métal sombre et statique) et une tenue de voix synthétiques, les cordes (traitées par informatique) jouent deux notes ré et $f a$ (croche deux doubles) répétées en miroir sur une cellule de base dans le médium-grave (la-fa-ré-la). Une autre ligne fait entendre une nappe la et sib au rythme instable, par dessus laquelle se rajoute une variante épique de cuivres qui entonnent la tierce mineure ascendante ré-fa, en valeurs longues, ponctuée par le rythme itératif et syncopé de la note ré (proche de la dance ou de la transe). Zimmer se démarque des blockbusters néo-hollywoodiens en intégrant cette tierce épique - et non plus « la quinte ascendante héroïque » (Huvet 2017, p. 80) - de forme interrogative et au rythme iambique (stagnance selon Fleury 1996, p. 231), suggérant la vulnérabilité de ce super-héros non doté de pouvoirs surnaturels, en proie aux doutes et souvent confronté à des choix difficiles, à ce "statisme généralisé [...] accru par l'emploi de polarisations parfois extrêmement longues " (Revol 2004, p. 55). Ce thème de Batman Begins sert visiblement de "patron " au morceau de Da Vinci code intitulé "Chevaliers de Sangreal ", apparaissant au moment où tous les éléments font sens pour le professeur Langdon, le menant, tel un élu (chevalier), au décryptage final après une longue quête difficile. Un battement numérique dans le grave sur lequel un motif en double (même si on passe en ternaire ici), ayant la même allure interrogative, forme un cycle amorcé par les mêmes notes autour de l'accord de ré mineur. Les harmonies changent davantage, mais elles sont non modulantes et contemplatives (modalité, accord parfait et septième d'espèce ${ }^{9}$ ). Au fur et à mesure (on conserve la même idée de gonflement pyramidal des couches sonores), le "lyrisme épique " (Carayol 2012, p. 60-65) des cordes, puis des cuivres synthétiques (doublés par les voix aiguës reliées au caractère sacré de l'intrigue), émanent progressivement de cette trame minimaliste au moment où le professeur trouve enfin l'emplacement du tombeau de Marie-Madeleine. Pour Interstellar, Zimmer a enregistré l'orgue de Temple Church à Londres (choisi pour son ampleur colossale) dont il a ensuite " samplé » chaque son en une infinité de cellules musicales de synthèse ("Cornfield Chase »), prenant de plus en plus de place dans le geste (humain) de la composition porté ici par le genre de la science fiction. La progression du thème pensée également par un épaississement de cellules qui se démultiplient ajoutant des strates au motif de base ( $f a \mathrm{M} 7$, sol6, lam, sol6/5), nourrit encore une fois une parenté - même si ce n'est pas aussi ostensible que pour Inception - avec celle de la Ligne rouge, partition matrice. Associés à d'autres sons réalisés à partir de machines (dub step, sub-bass, sound design), ces samples de l'orgue, auquel on a retiré à la fois son appartenance à une temporalité bien définie et

9 Détail de la succession d'harmonies du cycle ("Chevaliers de Sangreal ») : ré $\mathrm{m}$, ré chiffré 6/5, fa $\mathrm{M}$, do sus 4 , do $\mathrm{M}$, la $\mathrm{m}$. 
son essence " organique ${ }^{10}$, deviennent la catharsis d'une peur transcendée. Celle de ce groupe d'astronautes, à l'instar du personnage principal Cooper (père de famille), qui tente un voyage interstellaire par l'intermédiaire d'un trou de ver ${ }^{11}$ bouleversant totalement le temps du vécu terrestre au détriment de tout attachement familial. La mélodie qui émerge sur la trame, en forme de question (évoquant de ce point de vue la construction thématique de Batman et de Da Vinci code), fait entendre successivement et inlassablement une quinte (la-mi), une quarte (sol-mi), une tierce (do-mi) et une seconde (ré-mi) à l'image de l'étau qui se resserre pour ce père de famille et ses deux enfants qui voient leur espace-temps bouleversé - brouillé - lorsqu'il part à la conquête d'une planète viable qui pourrait permettre de préserver l'espèce humaine.

Certains motifs à la dimension contemplative de La Ligne rouge (le war adagio), Batman (le thème principal, fin du morceau" Lasiurus "), Da Vinci Code (" Malleus Maleficarum », "Chevaliers de Sangreal ») Inception ( Time ») et Interstellar ("Day one », "Cornfield Chase ») naissent et/ou s'éteignent sur un cliquetis électronique (oscillation d'une infrabasse, répétition ininterrompue d'une même note et autre battement d'ailes de chauve-souris mêlé à une rythmique pop) qui relève de "l'effet chronomètre ", lequel " est un moyen d'évoquer le décompte du temps, souvent à la manière de vieilles montres à aiguilles » (Brosch 2018, p. 55). Chez Zimmer, les sonorités numériques remplacent les "synthétiseurs imitant les cymbales charleston" pour lancer " un compte à rebours avant même que les notes du thème ne se fassent entendre " (ibid.). Elles soulignent ensuite les cellules répétitives ou donnent une pulsation quasi subliminale, mais suffisamment présente pour engendrer une sensation d'imminence, à ces trames étirées sur un temps long. Cet " effet chronomètre ", se conjuguant idéalement avec l'écriture minimaliste zimmerienne, permet de renforcer le caractère inéluctable de ces destinées sombres (fatalité et âpreté du combat dans La Ligne rouge), de ces quêtes inaccessibles ( $D a$ Vinci Code, Inception) ou d'exacerber le sentiment d'incertitude et la vulnérabilité du héros (Batman Begins), parfois confronté à des phénomènes qui le dépassent (Interstellar).

De manière plus générale, dans les films analysés ici, les nappes électro-minimalistes sont surtout le symbole d'un héroïsme hors du temps (Batman, Da Vinci Code) ou encore celui d'un éternel recommencement, brouillant les repères spatio-temporels (La Ligne rouge, Inception, Interstellar), où « temps et non-temps sont indissociablement liés » (Pärt 2010, au sujet de Trisagion) : elles se font l'écho du brouillard blanc qui enveloppe les soldats d'une menace ennemie invisible peu avant l'affrontement, de ces entrelacs du conscient et du subconscient dans lesquels Cobb est possiblement perdu, ou encore des imbrications parallèles de cet espace-temps (dilaté ou compressé) qui change fondamentalement la manière dont Cooper communique avec sa fille (afin qu'elle puisse l'aider à réussir sa mission).

10 Selon le compositeur, l'orgue « prend vie [...] par de grandes goulées d'air, comme nous les humains restons vivants grâce à la respiration » (propos recueillis par Rivaud 2014).

11 Un trou de ver (étudié dans le domaine de la physique quantique) est une sorte de sas sphérique de l'espace-temps qui permettrait d'atteindre une autre galaxie. 
Quelle retentisse en solo, en ostinato ou répétée sur un motif, la quinte, quintessence du minimalisme zimmerien (au-delà de l'accord parfait pärtien), symbolise l'instant suspendu.

Le film La Ligne rouge, marqué par l'esthétique contemplative de Malick, comporte naturellement l'une des premières manifestations de cette utilisation de la quinte dans la séquence où deux soldats se font tuer alors qu'ils partent faire un repérage dans les collines gardées par les japonais. Ils sont comme paralysés par la crainte du sort funeste que leur réserve leur progression vers le camp adverse. Ils sont figés dans l'instant suspendu d'une ellipse temporelle connotée à la fois par le changement de la lumière sur les herbes hautes de la colline balayée par le vent, ainsi que par la quinte à vide (ré-la) étendue à tous les registres de l'orchestre (résonant plus ostensiblement à la harpe). Selon Michel Fleury, les quintes superposées suscitent un statisme que différents compositeurs associent à l'idée de solitude, de calme immuable de la nature ou encore d'évocation de la torpeur moite de la jungle profonde (Fleury 1996, p. 220). Trois qualificatifs qui correspondent au ressenti des soldats dans le film et à l'atmosphère générale de l'intrigue (extrait vidéo 4).

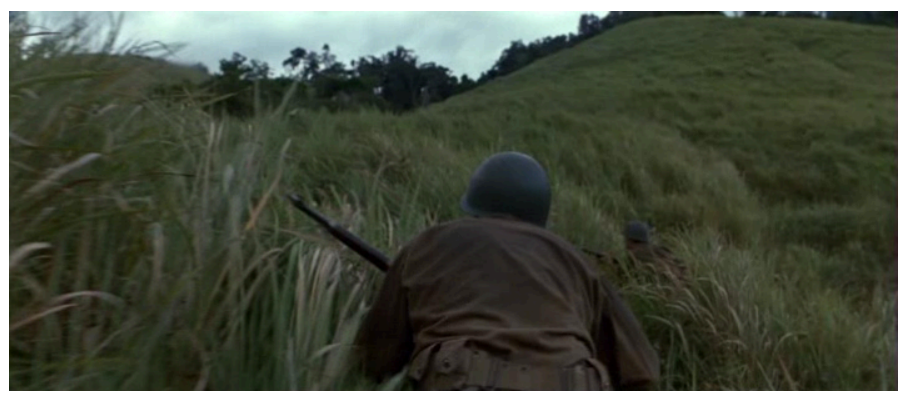

Extrait vidéo 4 : Terrence Malick, La Ligne rouge, scène où deux soldats partent faire un repérage dans les collines (00:44:36-00:45:17) (C) Twentieth Century Fox Film Corporation.

La courte scène précédant un des climax horrifiques du film Hannibal où le docteur cannibale se joue de sa proie, ici l'inspecteur italien Pazzi attaché à la verticale comme un judas crucifié, lui infligeant la torture suprême de suspendre le temps avant de le tuer, fait appel au même principe : une quinte ascendante est jouée en ostinato par la harpe sous le contrechant cristallin du piano - variation du motif d'Hannibal - et les réverbérations électroniques liées à l'enquête menée par Pazzi. Cette suspension poétique du temps par la musique crée, à l'image de la mise en scène dont l'action se déroule dans la ville de Florence, du beau dans l'horreur, ce qui accentuera d'autant plus la sauvagerie du meurtre qui va suivre au son d'une musique bruitiste faite de distorsions (extrait vidéo 5). Une quinte obstinée en doubles croches retentit aussi à la harpe sous le motif "Malleus Maleficarum " dans Da Vinci Code lorsque Sir Teabing explique le secret de la descendance du Christ. C'est encore une succession de quintes descendantes en marche (suivant un schéma tonal simple) qui apparait ostensiblement aux synthétiseurs dans Inception au moment où Cobb revient chez lui se demandant s'il va enfin pouvoir retrouver ses enfants - illusion ou réalité dans l'ambiguïté de cet instant suspendu. C'est enfin la note mi, ostinato suspensif, quinte de l'accord de la mineur autour duquel s'enroule la mélodie qui résonne dans 
Interstellar pour exprimer le doute et la fascination d'un père de famille dans l'instant suspendu d'une décision grave quand il se prépare à l'idée de piloter un vaisseau dans une autre galaxie (afin de potentiellement y trouver un endroit habitable alors que la terre se recouvre peu à peu d'une poussière mortelle) en sachant qu'il ne verra pas ses enfants grandir (extrait vidéo 6).

Extrait vidéo 5 : Ridley Scott, Hannibal, scène précédant le meurtre de Pazzi (01:12:40$01: 14: 17)$ (C) 2001 Métro-Goldwyn-Mayer Pictures Inc. et Universal Studios.
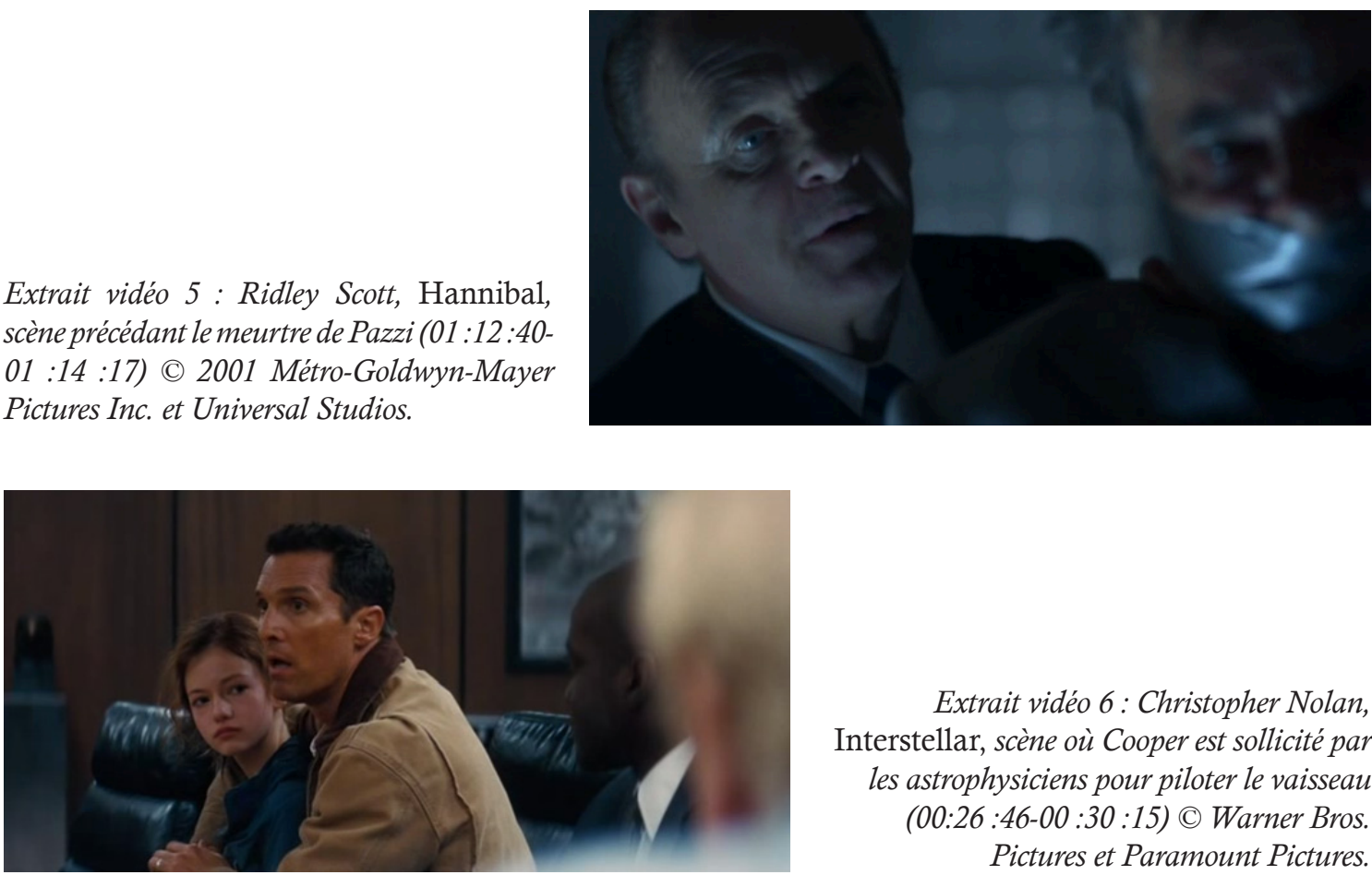

Extrait vidéo 6: Christopher Nolan, Interstellar, scène où Cooper est sollicité par les astrophysiciens pour piloter le vaisseau (00:26:46-00 :30 :15) (C) Warner Bros. Pictures et Paramount Pictures.

«Wormhole »-ANTI-LYRISME ET CLUSTER DIATONIQUE EN BLEND MODE : DÉSESPOIR, MORT ET NÉANT

L'écriture électro-minimaliste zimmerienne va parfois jusqu'à la négation de toute mélodie, remplacée par une note unique devenue texture abstraite ou encore par un cluster diatonique en blend mode afin d'évoquer le désespoir, la mort, ou le néant, dans un contexte narratif plus ou moins contemplatif.

Dans La Ligne rouge, la progression des soldats américains vers le camp ennemi dans les explosions, est accompagnée par une note tenue - sorte de râle dans l'extrême grave. Le caractère chaotique de la séquence naît de cet anti-lyrisme produisant une sorte de "gel dramatique ", lequel selon Mario Litwin ne peut être obtenu que par " un son tenu ou prolongé " et "impossible à obtenir avec une musique mouvante ou même un silence " (Litwin 1992, p. 57) - pratiquement à l'infini ici. Ce " presque rien " musical funeste rampe au côté des prises de vue à ras du sol (plans fixes ou travelling) qui accompagnent ces soldats et exacerbe le caractère impitoyable - parfois fatal - de ces explosifs (extrait vidéo 7). Si « drones, vrombrissement, infrabasses permanentes, etc. » sont « la quintessence du style Remote Control» (Berthomieu 2013, p. 693), le film de Malick a sans doute une valeur initiatique dans l'apparition de la sub-bass chère à Zimmer qui participe amplement à créer ce " gel dramatique " : cette sub-bass qui sert de toile de fond à la quasi totalité des motifs originaux de la partition (y compris le war adagio), se fait l'écho d'une destinée sombre tracée par 
la guerre, que rien - ni la dimension contemplative, ni la beauté de la nature, ni les réflexions intérieures - ne peut empêcher. Au sujet du traitement du son dans La Ligne rouge, Martin Barnier remarque que l'« on trouve ici assez peu d'infrabasses en comparaison avec la plupart des films de guerre contemporains. L'homme doit se taire devant la nature, rester contemplatif, seule sa pensée pouvant flotter sur les images » (Barnier 2007, p. 41-49). En prenant à son compte ce sound design que voulait éviter le cinéaste dans son discours narratif, Zimmer, à travers l'usage de la sub-bass, crée de nouveau une complémentarité oxymore avec les images afin de compenser ce qu'un bruitage trop lourd aurait altéré, tout en préservant le caractère en demi-teinte du film.

Dans Da Vinci Code, la première partie du cue "The Paschal Spiral " se compose d'un accord de la mineur joué par les cordes sur un mi de la contrebasse. Cet accord est altéré par une pédale électronique de si et un halo de synthèse faisant résonner un sol dans le lointain, puis il laisse place à un cluster diatonique (ré-mi-fa) du piano et des voix en blend mode, avant de réapparaitre. Cette partie du morceau amorce la scène où Silas (qui n'hésite pas à tuer pour accéder au secret gardé par le prieuré) est filmé comme un vampire : que ce soit le travelling spiralique ascendant nous menant à sa chambre plongée dans une demie obscurité rappelant l'ouverture d'Entretien avec un vampire (Neil Jordan, 1994), la lumière froide bleutée, sa toge noire, le fait qu'il soit albinos, la voix transformée du guide durant la conversation téléphonique en latin ou son ombre falote écrasée dans une sorte de faux raccord (figures $3 a$ et $3 b$ ). Silas est comme désincarné de lui-même (peu avant de mourir, ses derniers mots seront "Je suis un fantôme "). Le tout renvoie à sa dévotion absolue et extrêmement malsaine que la musique exprime ici avec un motif à la fois mystique (chaque partie ABA retentit trois fois - chiffre symbolique - la pédale électronique de si se rapproche d'un son de cloche) et sombre (anti-lyrisme, cluster et tremblement crescendo d'une infrabasse dans laquelle se loge un chœur d'hommes ${ }^{12}$ quasi imperceptible). Ce morceau associé à ce messager de la mort dans Da Vinci Code deviendra la genèse du motif intitulé "The Wormhole " (Trou de ver) dans Interstellar, notamment au moment où le vaisseau piloté par le héros est symboliquement happé par cet immense trou noir. Même si le timbre bruitiste des cordes qui souligne le mouvement en spirale de la caméra rappelle certaines partitions de science-fiction (Rencontres du troisième type, Spielberg/Williams, 1977 ; Alien, Scott/Goldsmith, 1979), la texture globale (piano, flûte alto, voix en blend mode, cordes) évoque celle de "The Paschal Spiral». On retrouve une même insistance sur la note mi (notamment la même pédale dans le grave), un même caractère lancinant, suspensif et quasiment le même cluster (do-rémi-fa). Ce motif, plus ou moins varié, est repris dans le film à chaque fois qu'il est question de se confronter aux aléas de l'espace, remettant sans cesse en cause la possibilité pour Cooper de retrouver sa famille et où, dans tous les cas, chaque minute écoulée l'éloigne spatio-temporellement des siens (extrait vidéo 8). Pour paraphraser Christophe Franco-Rogelio lorsqu'il analyse le Credo de Pärt intégrant le Prélude de

12 L'utilisation d'un chœur d'hommes bouche fermée ou aux paroles blasphématoires est devenu un topique du cinéma d'horreur (Carayol 2017b, p. 154). 
Bach dans son ouvrage Éthiques du temps (Franco-Rogelio 2014, p. 130), dans les deux films, cette scansion récurrente de ce cluster diatonique " entraîne " tous les autres motifs - ceux au lyrisme dissonant pärtien associé au mysticisme sombre de Silas ou encore les cellules de l'orgue reliant ailleurs Cooper à sa famille - vers lui « à la manière d'un trou noir ».

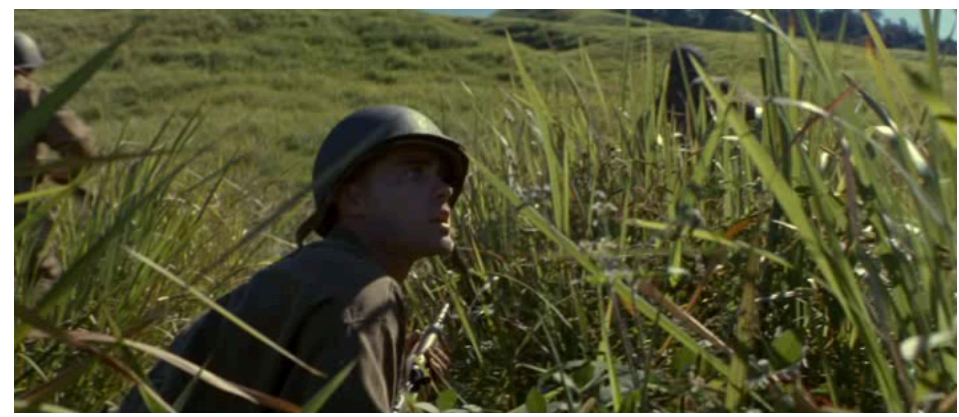

Extrait vidéo 7: Terrence Malick, La Ligne rouge, progression des soldats américains vers le camp ennemi dans les explosions (00:45:20-00:47:53)

(C) Twentieth Century Fox Film Corporation.

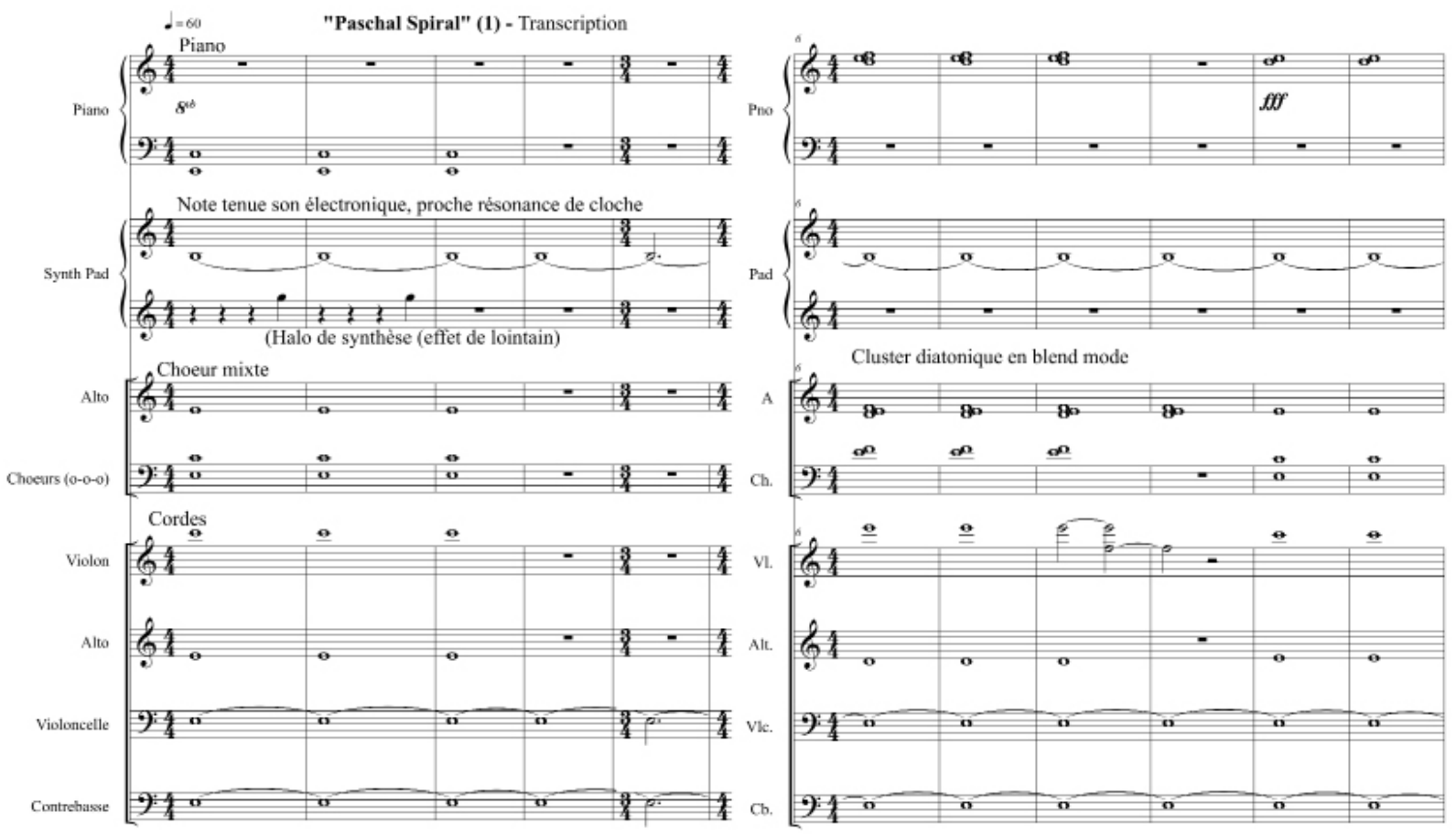

Exemple 7 : Hans Zimmer, Da Vinci code, cue "The Paschal Spiral».

Transcription personnelle à partir de la bande originale (00:00-00:19). 

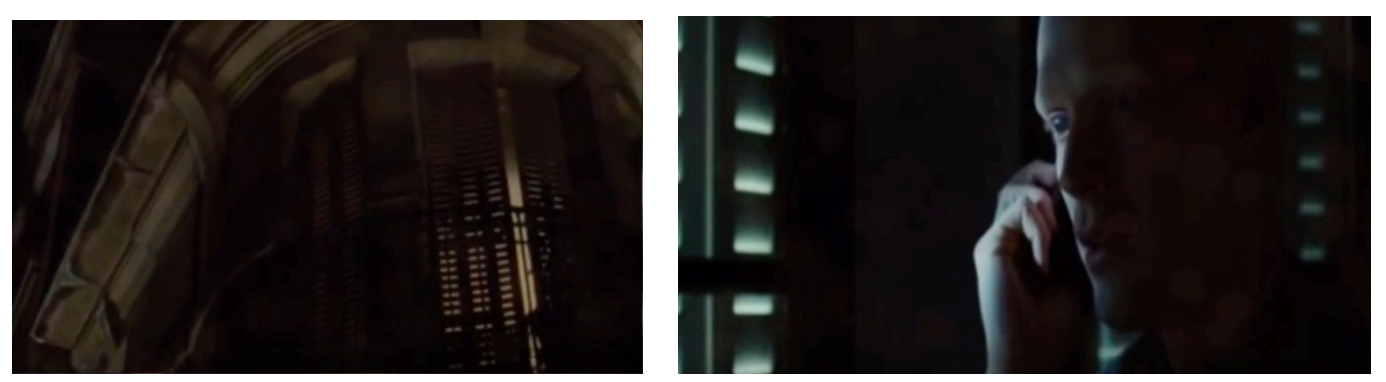

Figures $3 a$ et $3 b$ : Ron Howard, Da Vinci Code, deux photogrammes de la scène où Silas est assimilé à un vampire (06:19-07:15) (C) Colombia Pictures.

Extrait audio 6: Hans Zimmer, Da Vinci Code, cue "The Paschal Spiral» (00:00-00:54) (C) Decca. Écouter.

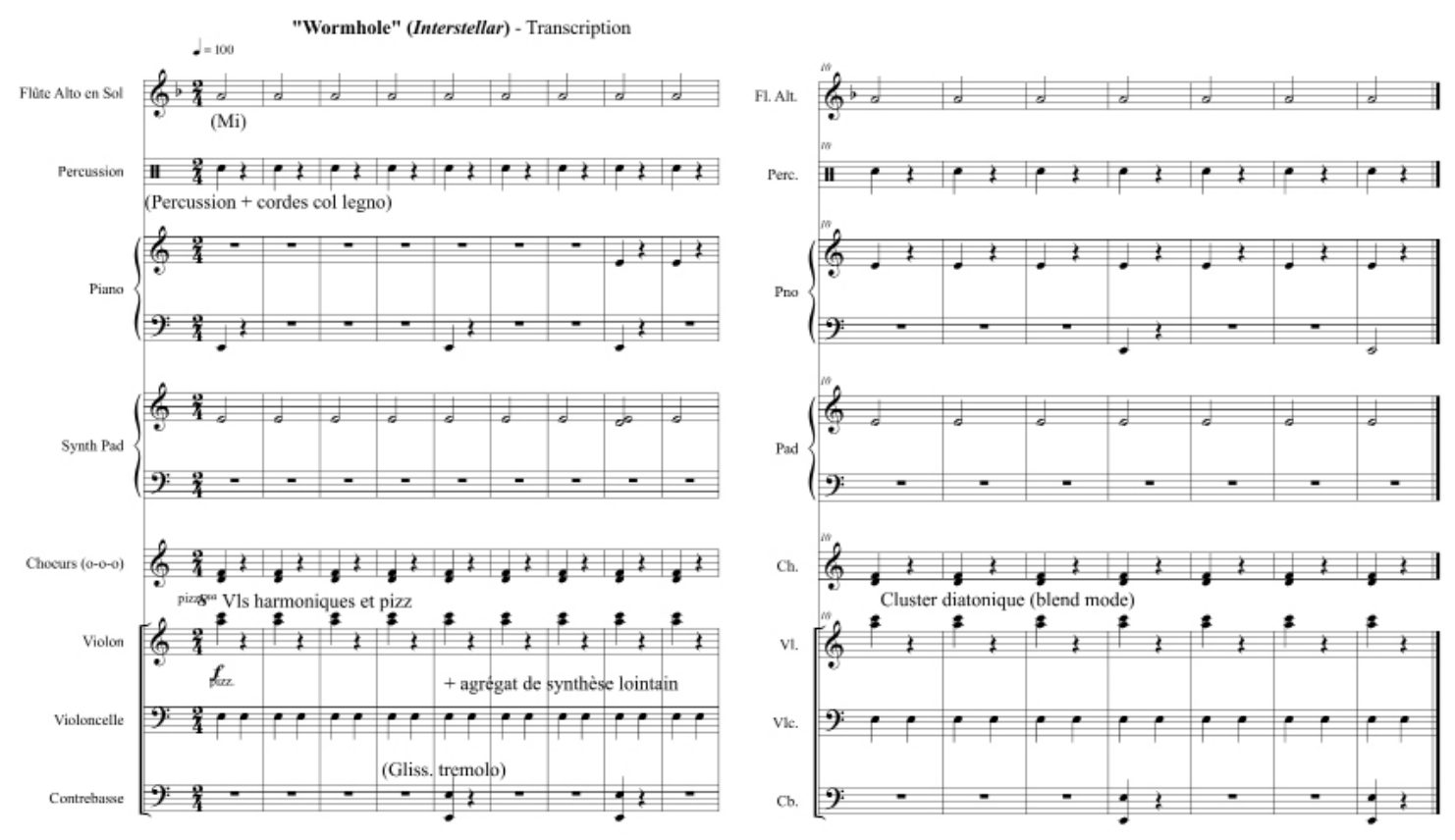

Exemple 8: Hans Zimmer, Interstellar, cue "The Wormhole 》. Transcription personnelle à partir de la bande originale (00:09-00:28).

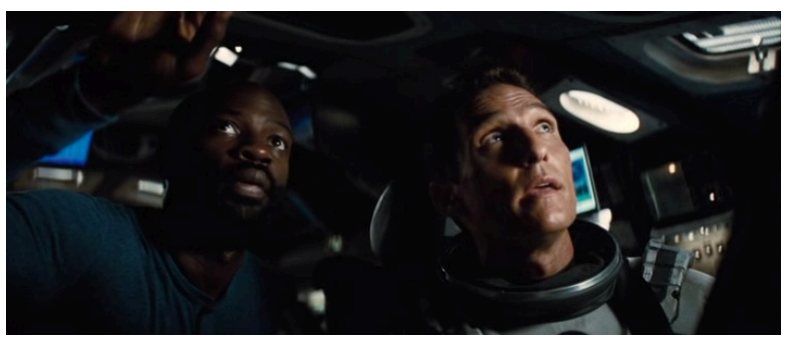

Extrait vidéo 8: Christopher Nolan, Interstellar, scène où le vaisseau piloté par Cooper s'apprête à pénétrer dans un trou de ver (00:56:33-00:58:09)

(C) Warner Bros. pictures et Paramount Pictures. 
CONCLUSION - «NEW MOON » : MINIMALISME DANS LES BLOCKBUSTERS HOLLYWOODIENS CONTEMPORAINS

Même si on ne peut évidemment pas réduire l'écriture de Zimmer à ce seul aspect ${ }^{13}$, il est tout de même possible d'affirmer que ce minimalisme, référence ciblée à Arvo Pärt (plus rare au cinéma que celle de John Adams et Philip Glass) ou plus globale, mêlée à cette énergie créative de timbres hybrides, depuis la partition initiatique $\mathrm{La}$ Ligne rouge, atteste bien d'une appréhension nouvelle du rapport musique-image : que ce soit cette temporalité inéluctable élaborée à partir des nappes électro-minimalistes, la quinte - sorte de tintinnabulum zimmerien - soulignant l'instant suspendu ou cette forme de radicalisation qui s'exprime dans l'emploi de clusters diatoniques en blend mode pour évoquer la mort et le néant. Ce schème minimalisme-blockbuster, sur lequel Berthomieu insiste dans ses écrits récents (Berthomieu 2013, p. 697-698) au sujet de films où les caractères épique et héroïque dominent et auxquels on n'associerait moins naturellement ce type d'écriture (cette remarque étant à nuancer au regard d'un film comme La Ligne rouge où l'esthétique de Malick invite la dimension contemplative) peut sans doute être considéré comme une force vive et originale de cette empreinte musico-sonore hollywoodienne contemporaine :

En quête de la note parfaite pour créer le son du Joker de The Dark Knight, le discours d'Hans Zimmer se calque presque sur l'ambition théorique de Pärt. [...] Dans la trilogie de Batman, le héros est signalé par deux notes ré-fa et le Joker s'annonce par la manipulation électronique d'une seule note suraiguë (un ré) qui en devient deux (ré-fa aussi) sous forme de palpitation synthétique. [...] Man of Steel répète le principe du Dark Knight : la formule musicale de Superman se résume à deux notes, une quinte en do majeur, une sorte de début de fanfare à la Copland/Williams en version minimale, répétée en boucle (ibid., p. 697-698).

Et plus loin, il ajoute : « Da Vinci code reste à ce jour la synthèse la plus raffinée des influences minimalistes chez Zimmer, de Penderecki à Pärt " (ibid.).

À l'instar de ce phénomène qu'Hans Zimmer (et Remote control) participe activement à instaurer, un compositeur comme Alexandre Desplat qui est issu du symphonisme intimiste (courant du cinéma français contemporain ${ }^{14}$ ) et qui s'inspire également d'Arvo Pärt ${ }^{15}$, notamment dans ses partitions pour Jacques Audiard, transpose ce type d'écriture, par lequel il est imprégné depuis ses débuts, dans les

\footnotetext{
13 Pour chaque film, Zimmer, qui a un sens aiguisé de l'image, cherche un concept visant à cibler au plus juste le propos narratif : dans Da Vinci code, par exemple, le compositeur repense le leitmotiv avec une approche stematique - par stem (procédé de mixage, sous Pro Tools par exemple, qui permet de masquer certaines pistes instrumentales) - guidée par sa virtuosité dans la MAO. C'est à dire qu'il entremêle des motifs ou des nappes sous forme de strates (verticalement et/ou horizontalement) dans une infinité de combinaisons afin de créer un réseau thématique dense à l'image de ce jeu de décryptage que comporte l'intrigue.

14 Voir à ce sujet Carayol 2012.

15 «Parmi les compositeurs du symphonisme intimiste, Alexandre Desplat est celui qui va le plus loin dans l'expérimentation du minimalisme au cinéma dans des films comme Sur mes lèvres, De battre mon coeur s'est arrêté et Les Corps impatients. [...] En appliquant également son écriture minimaliste et épurée à tous les paramètres (mélodie, harmonie, timbre, rythme, dynamique), Alexandre Desplat crée un minimalisme intégral » (ibid., p. 129).
} 
films anglo-saxons et américains. Une des singularités de Desplat - et c'est sans doute une des raisons pour laquelle il est autant sollicité, c'est qu'il utilise aussi bien le minimalisme dans des films intimistes (où ce langage est devenu une convention du genre $^{16}$ ) comme La Jeune Fille à la perle (Peter Webber, 2003), Bright Star (Jane Campion, 2009), The Tree of life (Terrence Malick, 2011) ou encore The Danish Girl (Tom Hooper, 2015), que dans des blockbusters, et en ce point il rejoint Zimmer, mais avec son style propre $^{17}$, comme dans les sagas Harry Potter et les reliques de la mort (David Yates, 2010 et 2011) ${ }^{18}$, ou encore Twilight : New Moon (Chris Weitz, 2009). Dans ce dernier film, par exemple, il peut passer d'un langage proche du symphonisme intimiste dans des cues destinés à des scènes sentimentales comme "Memories of Edward ", "The Cullens ", ou "Marry Me, Bella " à une forme "d'undersoundesigning ${ }^{19}$ " (Carayol 2017a) pour les scènes d'action ("Wolves V. Vampire », " The Volturi »), comme le fait ailleurs Hans Zimmer, même si c'est de manière moins texturale, moins abstraite et plus symphonique (en ce sens Desplat propose une alternative stylistique située à mi-chemin entre néo-hollywoodisme et Remote Control).

Ainsi, la musique du cinéma hollywoodienne contemporaine, portée en partie par l'école Zimmer à l'initiative d'une esthétique oxymore mêlant minimalisme, épure et machines massives, que l'on ne peut plus réduire désormais « au vide d'un présent sans rêve » (Berthomieu 2004, p. 75), offre un renouveau nécessaire, induit notamment par les avancées techniques dans le domaine des effets sonores d'un film, menant des compositeurs néo-hollywoodiens (comme James Horner ${ }^{20}$, Danny Elfman ou John Williams) - hors Remote control - à repenser leur approche d'une musique de film dans leurs bandes originales les plus récentes ${ }^{21}$.

\section{BIBLIOGRAPHIE}

Barnier, Martin (2007), "Le cri de la nature chez Terrence Malick. Analyse du son de The Thin Red Line », Décadrages. Cinéma à travers champs, nº 11 : « Terrence Malick », p. 41-49.

Bellin, Joshua David (2005), Framing Monsters, Fantasy Film and Social Alienation, Southern Illinois University Press.

16 La Leçon de piano (Jane Campion, Michael Nyman, 1993), Eyes Wide Shut (Stanley Kubrick, Jocelyn Pook, 1998), The Hours (Stephen Daldry, Philip Glass, 2002), Carol (Todd Haynes, Carter Burwell, 2015).

17 Écriture plus symphonique, utilisation d'instruments exotiques ou modes de jeux inusités.

18 Voir Pignolet 2018.

19 «L'undersoundesigning (stricte transposition moderne du concept d'underscoring évoqué notamment par Kalinak) consiste à créer une orchestration symphonique et/ou hybride qui puisse rivaliser avec le sound design (et non plus le dialogue, problématique déplacée) aux infrabasses de plus en plus marquées, par l'utilisation de percussions conventionnelles aux côtés de percussions très résonnantes telles que le taiko japonais (percussion le plus souvent jouée en polyrythmie par 3 - petite, médium et grande), l'emploi de certains pupitres repris par l'overdub (servant à démultiplier le timbre numériquement) ou d'un cuivre solo renforcé par le mixage. Le tout pensé dans un juste équilibre afin de ne pas générer une valeur ajoutée qui viendrait créer un chaos sonore tonitruant, mais qui comporte néanmoins un niveau sonore suffisant pour s'entremêler en contrepoint timbral au sound design qui accompagne les scènes d'action » (Carayol 2017a).

20 Compositeur né en 1953 et mort en 2015.

21 Voir à ce sujet Huvet 2017. 
Berthomieu, Pierre (2004), La musique de film, Paris, Klincksieck.

Berthomieu, Pierre (2013), Hollywood. Le temps des mutants, Pertuis, Rouge Profond.

Brosch, Ludwig (2018), La musique des films d'épouvante aux États-Unis dans les années 1970. Une décennie du renouveau?, mémoire de master, Université de Rouen.

Carayol, Cécile (2012), Une musique pour l'image, vers un symphonisme intimiste dans le cinéma français, Rennes, Presses Universitaires de Rennes.

Carayol, Cécile (2015), «Dimension contemplative et minimaliste de la musique dans le film de guerre : exemple de La Ligne Rouge (Terrence Malick, Hans Zimmer, 1998) », communication lors de la journée d'étude dirigée par Chloé Huvet et Jérémy Michot, Reconfiguration des pratiques musicales dans le cinéma hollywoodien contemporain, Université Rennes 2, 28 novembre 2015

Carayol, Cécile (2017a), "Effets spéciaux de l'orchestre dans Twilight, New Moon (Weitz-Desplat, 2009) : modes de jeux et alliages instrumentaux spécifiques d'un épique obscur entre tradition et renouveau ", communication lors de la journée d'étude dirigée par Jérôme Rossi, L'orchestre et la musique de film, Université de Nantes, 22 septembre 2017. Disponible en ligne : https://webtv. univ-nantes.fr/fiche/10859/cecile-carayol-effets-speciaux-de-l-orchestre-dans-twilight-new-moonweitz-desplat-2009-modes-de-jeux-et-alliages-instrumentaux-specifiques-d-un-epique-obscurentre-tradition-et-renouveau.

Carayol, Cécile (2017b), «La figure du vampire : codes récurrents dans la musique symphonique de film de la Hammer à Twilight (New moon)», dans Cécile Carayol, Pierre-Albert Castanet et Pistone Pascal (dir.), Le fantastique dans les musiques des XX $X^{e}$ et XXI e siècles, Sampzon, Delatour France, p. 145-178.

Caux, Daniel (2004), «Arvo Pärt. L'art du «tintinnabulum» », L'éducation musicale (septembre/octobre), p. $52-53$.

Chion, Michel (1995), La musique au cinéma, Paris, Fayard.

Chion, Michel (2004), La Ligne rouge, Paris, La Transparence/Cinéphilie.

Fleury, Michel (1996), L'impressionnisme et la musique, Paris, Fayard.

Franco-Rogelio (2014), Christophe, Arvo Pärt/Philip K Dick. Éthiques du temps, Paris, Camion blanc.

Guiomar, Michel (1988), Principes d'une esthétique de la mort, Paris, José Corti.

Huvet, Chloé (2017), D’Un nouvel espoir (1977) à La Revanche des Sith (2005). Écriture musicale et traitement de la partition au sein du complexe audio-visuel dans la saga Star Wars, thèse de doctorat, Université Rennes 2 et Université de Montréal.

Jankélévitch, Vladimir (1976), Debussy et le mystère de l'instant, Paris, Plon.

Litwin, Mario (1992), Le film et sa musique : création, montage, Paris, Romillat.

Moindrot, Gérard (2004), "Cantus in memoriam benjamin Britten (1977) », L'éducation musicale (septembre/octobre), p. 69-72

Pärt, Arvo (2010), dans "Arvo Pärt, la musique qui tintinnabule », Esprits nomades (31 mars), http://www.espritsnomades.com/siteclassique/arvopart.html, consulté le 22 mai 2018

Pignolet, Sébastien (2018), Le minimalisme d'Alexandre Desplat dans le cinéma hollywoodien contemplatif et épique : exemples de The Tree of life (T. Malick, 2011) et Harry Potter et les Reliques de la mort (D. Yates, 2010-2011), mémoire de master, Université de Rouen.

Revol, Patrick (2004), « Miserere d'Arvo Pärt », L'éducation musicale (septembre/octobre), p. 54-68.

Rivaud, Sylvain (2014), " Christopher Nolan et Hans Zimmer racontent Interstellar. Décryptage avec témoignages ", Cinezic (23 novembre), http://www.cinezik.org/infos/affinfo. php?titre $0=20141123203223$, consulté le 28 mai 2018. 